\title{
What happened before?
}

\section{The disks around the precursors of young Herbig Ae/Be stars.}

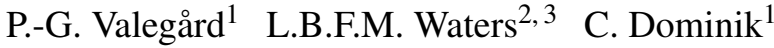 \\ 1 Anton Pannekoek Institute for Astronomy (API), University of Amsterdam, Science park 904, 1098 XH Amsterdam, The Nether- \\ lands \\ e-mail: p.g.valegard@uva.nl \\ 2 Institute for Mathematics, Astrophysics \& Particle Physics, Radboud University, P.O. Box 9010, MC 62, NL-6500 GL Nijmegen, \\ the Netherlands \\ 3 SRON, Sorbonnelaan 2, 3484CA Utrecht, The Nederlands
}

Received October 30, 2020; accepted April 15, 2021

\section{ABSTRACT}

Context. Planets form in circumstellar disks around pre-main sequence stars. A key question is how the formation and evolution of protoplanetary disks depends on stellar mass. Studies of circumstellar disks at infrared and submm wavelengths around intermediate mass Herbig Ae/Be stars have revealed disks structures such as cavities, gaps and spiral arms. The Herbig Ae/Be stars represent an older population of intermediate mass pre-main sequence stars. Since intermediate mass pre-main sequence stars evolve towards the main sequence on timescales comparable to typical disk dissipation timescales, a full picture of disk dispersal in intermediate mass pre-main sequence stars must include the intermediate mass T-Tauri stars.

Aims. We seek to find the precursors of the Herbig Ae/Be stars in the solar vicinity within $500 \mathrm{pc}$ from the Sun. We do this by creating an optically selected sample of intermediate mass T-Tauri stars (IMTT stars) here defined as stars of masses $1.5 M_{\odot} \leq M_{*} \leq 5 M_{\odot}$ and spectral type between $\mathrm{F}$ and $\mathrm{K} 3$, from literature.

Methods. We use literature optical photometry $(0.4-1.25 \mu \mathrm{m})$ and distances determined from Gaia DR2 parallax measurements together with Kurucz stellar model spectra to place the stars in a HR-diagram. With Siess evolutionary tracks we identify intermediate mass T-Tauri stars from literature and derive masses and ages. We use Spitzer spectra to classify the disks around the stars into Meeus Group I and Group II disks based on their $\left[\mathrm{F}_{30} / \mathrm{F}_{13.5}\right]$ spectral index. We also examine the $10 \mu \mathrm{m}$ silicate dust grain emission and identify emission from Polycyclic Aromatic Hydrocarbons (PAH). From this we build a qualitative picture of the disks around the intermediate mass T-Tauri stars and compare this with available spatially resolved images at infrared and at sub-millimeter wavelengths to confirm our classification.

Results. We find 49 intermediate mass T-Tauri stars with infrared excess. The identified disks are similar to the older Herbig Ae/Be stars in disk geometries and silicate dust grain population. The detection frequency of PAHs is higher than from disks around lower mass T-Tauri stars but less frequent than from Herbig Ae/Be disks. Spatially resolved images at infra-red and sub-mm wavelengths suggest gaps and spirals are also present around the younger precursors to the Herbig Ae/Be stars.

Conclusions. Comparing the timescale of stellar evolution towards the main sequence and current models of protoplanetary disk evolution the similarity between Herbig Ae/Be stars and the intermediate mass T-Tauri stars points towards an evolution of Group I and Group II disks that are disconnected, and that they represent two different evolutionary paths.

Key words. Protoplanetary disks - Stars:Evolution - Stars: Variables: T Tauri, Herbig Ae/Be - Stars: statistics - Stars: pre-main sequence

\section{Introduction}

Planets are formed in circumstellar disks around young pre-main sequence stars (PMS stars) or perhaps, even in the earlier protostellar phase (Segura-Cox et al. 2020). Angular momentum conservation during the collapse of the molecular cloud around the proto-stellar core forces material to form a disk around the forming star. The disk material dissipates over a few Myrs as the material is accreted onto the star, lost into space by disk winds and/or jets, and accreted into planets. The circumstellar disk evolves from a gas rich disk into a transitional disk and eventually a gas poor debris disk. This general scenario has gained substantial observational support over the last 10 years, as more spatially resolved observations around PMS stars have become available. Observations at $\mathrm{mm}$ and sub-mm wavelengths with instruments such as for example ALMA, SPHERE, GPI and SUB-
ARU have revealed circumstellar disks with spiral arms, central cavities, concentric circular gaps and warps in the disk indicative of interactions with planetary bodies (Andrews 2020a. Garufi et al. 2017; Andrews et al. 2018; Huang et al. 2018a b, Garufi et al. |2020; Benisty et al. 2018 ; Monnier et al.|2019). Other explanations for these features have also been suggested such as gravitational instabilities, snow lines and binary interactions (Andrews 2020b; van der Marel et al. 2019).

A key questions is to understand how the formation and evolution of planetary systems depends on stellar mass. Can we connect the properties of planet-forming disks to those of mature planetary systems, and what role does the stellar mass play? When do the features seen in observations of planetary disks, spirals, gaps and cavities, form and what do they say about the planetary formation process? 
Two main types of PMS stars have traditionally been observed to study the circumstellar disks and their evolution. The T-Tauri stars (spectral class $\mathrm{F}$ and later) (Joy 1945, Herbig 1962; Bastian et al. 1983) and the Herbig Ae/Be stars (hereafter HAeBe, spectral class B and A) (Herbig 1960, Bastian et al. 1983). Overlapping in mass, the T Tauri stars with masses $M \geq 1.5 M_{\odot}$ will evolve through the Hertzsprung-Russel diagram (HR-diagram) to become HAeBe stars. The studies of circumstellar disks using HAeBe stars only, however, have introduced a bias in our view of planet formation and disk dissipation in this mass range (see also Vioque et al. 2018).

In order to have a full observational view of disk evolution and planet formation for intermediate mass stars, samples must be constructed that contain both HAeBe stars and their precursors. Several papers have mentioned these T Tauri stars as a separate group. Herbig \& Bell (1988) used a special category called 'su' T Tau stars, defined as stars with a spectral appearance similar to the T Tauri star SU Aur. Herbst et al. (1994) used the term Early Type T Tauri stars or ETTS, containing both HAeBe stars and T Tauri stars. To our knowledge Calvet et al. (2004) was the first paper dedicated to and naming the intermediate mass $\mathrm{T}$ Tauri stars (hereafter IMTT stars). Lavail et al. (2017) studied magnetic fields in IMTT stars and defined the class as PMS stars with masses $1 M_{\odot} \leq M \leq 4 M_{\odot}$. These papers contain a relatively small number of objects and uses different mass ranges. The IMTT stars have so far been discussed as a part of a much larger samples of T Tauri stars. For the HAeBe stars, samples exists and are widely studied (e.g. Herbig 1960; The et al. 1994; Acke \& van den Ancker 2006; Juhász et al. 2010; Garufi et al. 2017; Varga et al 2018a; Vioque et al 2018).

It is therefore useful to construct a sample of IMTT stars from existing literature and study their properties, so that a more complete picture of planetary formation and disk evolution for intermediate mass PMS stars can be constructed and compared to the lower mass stars.

In this paper we have identified IMTT stars by searching the literature for luminous $\mathrm{T}$ Tauri stars in the solar neighborhood that may be of intermediate mass, defined by their mass $1.5 M_{\odot} \leq M \leq 5 M_{\odot}$ and spectral type later than $\mathrm{F}$. We construct spectral energy distributions (SEDs) and use literature values of spectral types and effective temperatures, together with Gaia DR2 data in order to place the stars into the HR diagram. We discuss the properties of the stars and their disks in the context of what is known for the HAeBe stars by examining disk geometry, silicate dust grains and Polycyclic Aromatic Hydrocarbons (PAH). Meeus et al. (2001) used the shape of the infrared and millimeter spectrum to classify the protoplanetary disks around HAeBe stars into two groups. In Group I (transitional disks) and Group II based on their SED. Since then many papers have used this classification and noted differences between the two groups. In this paper we will also use the Meeus classification so that a meaningful comparison with the HAeBe literature can be made.

This paper is organized as follows: In Section 2 we discuss the construction and refining of the sample. In section 3 we describe our analysis of the disk properties, focusing on spatially unresolved data. Section 4 discusses the properties of IMTT disks in the context of disk evolution and dissipation in intermediate mass PMS stars, and in Section 5 we summarize the main conclusions of our study.

\section{Sample selection}

\subsection{Approach}

The optically selected sample has been constructed by searching literature for IMTT stars in the solar neighbourhood. The selection procedure takes place in 4 steps. First an initial search for T-Tauri stars in the SIMBAD database. Then in a second step we remove stars that lie beyond a chosen distance maximum. We use a simple method to obtain a first estimate of luminosity in the third step in order to remove most of the low mass T-Tauri stars $\left(<1.5 M_{\odot}\right)$. Finally, in a forth step, the luminosity of the remaining stars are then determined more precisely by fitting a Kurucz stellar model to the photometry, allowing an accurate placement in the HR-diagram.

\subsection{Initial selection of stars from literature}

We searched the SIMBAD database for T-Tauri stars (keyword TT*) with spectral types between $F 0-K 3$. Earlier type stars one would contaminate the sample by including the Herbig stars; with later type stars than K3 estimating the mass correctly is challenging with our methodology because of the proximity of the pre-main sequence evolutionary tracks which run close together and are almost vertical in the particular luminosity range we are interested in. The search turned up 623 stars. We have also included HAeBe stars of spectral type $F$ and $G$ from Vioque et al. (2018) (2 stars) as well as the IMTT stars in the work by Villebrun et al.(2019) (14 stars) and Calvet et al.(2004) (2 stars).

\subsection{Distance determination and distance limit}

The disks around the stars should be observable in scattered light in the hope that disk features such as gaps, spirals and shadows in the disk can be observed. Gaps in the disk typically have an average width of $\leq 10 A U$ (Andrews et al.2018). With the current resolution of scattered light instruments such as VLT-SPHERE, that have a resolution limit of 22-27 mas (Beuzit et al. 2019) these features would not be easily detected at distances beyond $450 \mathrm{pc}$. Since disks themselves can be detected in scattered light at greater distances and the number of high mass star-forming regions in the vicinity of the sun is low we decided to include the Orion star forming region. Therefore we extend the distance limit to $500 \mathrm{pc}$, keeping in mind that features in the most distant disks will be difficult to resolve. In the second step, we obtained the distances to each star by bayesian inference from Gaia DR2 parallaxes by Bailer-Jones et al. (2018). This is an important asset for our method determining stellar parameters because of the high precision in the parallaxes measured by Gaia. The distance uncertainties by Bayesian inference presented in Bailer-Jones et al. (2018) for the final sample of stars are typically between $2-4 \%$. The uncertainties in luminosity for the stars is therefore dominated by the uncertainty in the determined $T_{\text {eff }}$. If no distance was available we used Hipparcos parallaxes. For stars where none of these options where available we searched literature for a distance measurement or estimate using associations of stars. Stars where no distance reference could be found or that where further away then $500 \mathrm{pc}$ where then removed from the sample. We have used all Gaia parallaxes in our selection steps, i.e. also those with RUWE above 1.4. We will come back to this point in section 2.5 when discussing the final sample. 


\subsection{Removal of lower mass T-Tauri stars}

The third step in the selection process aims to remove the bulk of the low mass T-Tauri stars. We first look for an accurate spectral class classification in literature or an accurate determination of $T_{\text {eff. }}$ Stars that have a classification as FU Ori stars were immediately removed because of their high variability from outbursts and because the flux at optical wavelengths are dominated by their accretion disks.

Pre-main sequence evolutionary tracks partially overlap with post-main sequence tracks. Stars identified as post-main sequence in the literature in this step were therefore removed from the sample. The remaining number of post-main sequence stars in our sample should then be very small. Infrared excess is not expected for single intermediate mass stars that have evolved to sub-giants, as these stars do not yet have dusty stellar winds.

We then collected optical B and V-band photometry using catalogs with good coverage. These catalogs included: NOMAD (Zacharias et al. 2005), UCAC4 (Zacharias et al. 2012), APASS DR2 (Henden et al. 2016), UBVRIJKLMNH Photoelectric cata$\log$ (Morel \& Magnenat 1978), Tycho2 catalog (Hog et al. 2000), EPIC (Huber et al. 2017) and Manoj et al. (2006). Using the table of standard stars from Pecaut \& Mamajek (2013) we obtain the intrinsic (B-V) for each spectral class and temperature as well as the bolometric corrections (BC) in V-band, $\mathrm{BC}_{V}$. Assuming an $R_{v}=3.1$ we estimated the extinction, $A_{v}$ and the luminosity from the V-band magnitude using the distance modulus. We then set a lower luminosity limit of $2.1 L_{\odot}$ based on the lowest luminosity of a Siess et al. (2000) stellar evolutionary track for a star of 1.5 $M_{\odot}$ with solar type metallicity $(\mathrm{Z}=0.01)$. Stars that are likely to be low mass T-Tauri stars are in this way removed. This cut the sample to 122 candidates.

\subsection{Final selection}

The fourth and final step in our source selection procedure involves placing the stars accurately in the HR diagram by means of SED fitting. In order to do so the extinction and luminosity needs to be accurately determined. Together with $T_{\text {eff }}$ the mass can be determined from evolutionary tracks in the HR diagram. Photometry used in this process covered $0.4-1.25 \mu \mathrm{m}$ to avoid excess from accretion in the UV and infrared excess from circumstellar material.

We used Gaia DR2 G-band photometry to examined the relative error in magnitude from the Gaia database (Gaia Collaboration et al. 2018) and conclude that most stars have low to moderate variability (at least during the Gaia measurement period) of typically around $1 \%$ with a few exceptions up to around $6 \%$. Some variability has to be expected because of the presence of possibly occulting circumstellar material. Therefore, we use the maximum measured brightness at each wavelength.

A Kurucz model spectrum was fitted to the optical photometry using the iterative method of Woitke et al. (2016) (see Appendix $\mathrm{A}$ in said paper). The method requires the following starting input parameters: $M_{*}$, distance, $T_{\text {eff }}, L_{*}, R_{v}$ and $A_{v}$. We use (as derived in previous subsection) the estimates for $A_{v}, L_{*}$ and the literature values for $T_{\text {eff }}$. The initial $M_{*}$ was set to $2 M_{\odot}$ and the $R_{v}=3.1$ for all stars (except 3 highly reddened sources, EM*SR 21, Haro $1-6$ and $\mathrm{LkH} \alpha 310$, were $R_{v}=5$ in the end gave a better stellar photospheric fit). The routine starts with calculating a $\log (g)$ assuming said starting parameters. This $\log (g)$ value is used to choose a Kurucz stellar spectrum. The spectrum is then reddened and fitted to the observed photometry, convolving the model spectrum with the filter transmission curves for each of the photometric bands to produce the integrated model flux in each band (Woitke et al. 2019). The resulting $L_{*}$ and $A_{v}$ is then fed back into the program, keeping $T_{\text {eff }}$ fixed. The stellar mass is refined for each iteration by deriving the value using Siess et al. (2000) pre-main sequence stellar evolution models. This process leads to a more accurate value $\log (g)$ and therefore a better model spectrum selection for the following iteration. The routine converges very quickly (typically in 2-3 iterations) to determine $M_{*}, L_{*}$ and $E(B-V)$. 1 We run this procedure not only for the $T_{\text {eff }}$ value, but also for the $T_{\text {eff }}$ range allowed by the uncertainty, in order to derive the corresponding range in luminosity.

Stars that were found to be below $1.5 M_{\odot}$ or were no fit could be made with the available photometry were removed. We also remove stars that have no infrared excess, i.e. the photometry follows the expected stellar photospheric emission. We do not apply a strict upper bound for the luminosity as a selection criterion.

Some of the Gaia parallaxes in our final sample are less reliable. We consider the parallaxes with re-normalized unit weight error, RUWE $\leq 1.4$ as 'good' 2 As a final step in our procedure, we therefore carefully check the distances for 10 stars in our final sample for which the Gaia RUWE was found to be $>1.4$. In appendix A a comparison is presented between the Gaia distance of these 10 stars and other distance determinations in the literature. Appendix A also presents our final value adopted for the distances of these stars.

A few notes on specific targets are on order:

- Two stars in the sample are classified as late type A-stars (HD 142666 and HD 144432). They have remained in the sample because their uncertainty in $T_{\text {eff }}$ overlaps with our sample criteria.

- We note that the $70 \mu \mathrm{m}$ and sub-mm fluxes measured for HBC 502 (Flaherty \& Muzerolle 2008, Mitchell et al. 2001) are very high. Muzerolle et al. (2010) suggest that this may be due a spatial coincidence with a class 0/I object. However, the optical and IR photometry is dominated by the IMTT star and therefore we kept this object in our list.

- For UX Tau we were unable to fit a model spectra. We adopted stellar parameters from literature (Akeson et al. 2019; Csépány et al. 2017, Espaillat et al. 2010, Kraus \& Hillenbrand 2009). Using these values we have kept it in the analysis because of its position in the HR-diagram.

- We also remove BP Psc, which is discussed to maybe be a T-Tau star $(\sim 80 \mathrm{pc})$ or a post-MS giant $(\sim 300 \mathrm{pc})$ (de Boer et al. 2017). The distance from Bailer-Jones et al. (2018) release puts it at $\sim 360$ pc suggesting a post main-sequence nature.

\subsection{Final sample}

We find 49 sources matching our selection criteria for IMTT stars, that also show evidence of infrared excess. An overview of the sample can be seen in table 1 together with the literature reference for their spectral class. The HR-diagram for the full sample is presented in figure 1 . The majority of the IMTT stars found are located in the Orion star forming complex. Other regions include Cepheus, Chameleon, Lupus, Ophiucus, Perseus

\footnotetext{
1 We found that for HD 34700 the iteration finished with a negative extinction, it was kept in the sample by adjusting the spectral type by a half subclass (i.e. we re-run the fit using a spectral type of F9 from Pecaut \& Mamajek 2013)

2 see technical note GAIA-C3-TN-LU-LL-124-01, in the Gaia DR2 release documentation.
} 


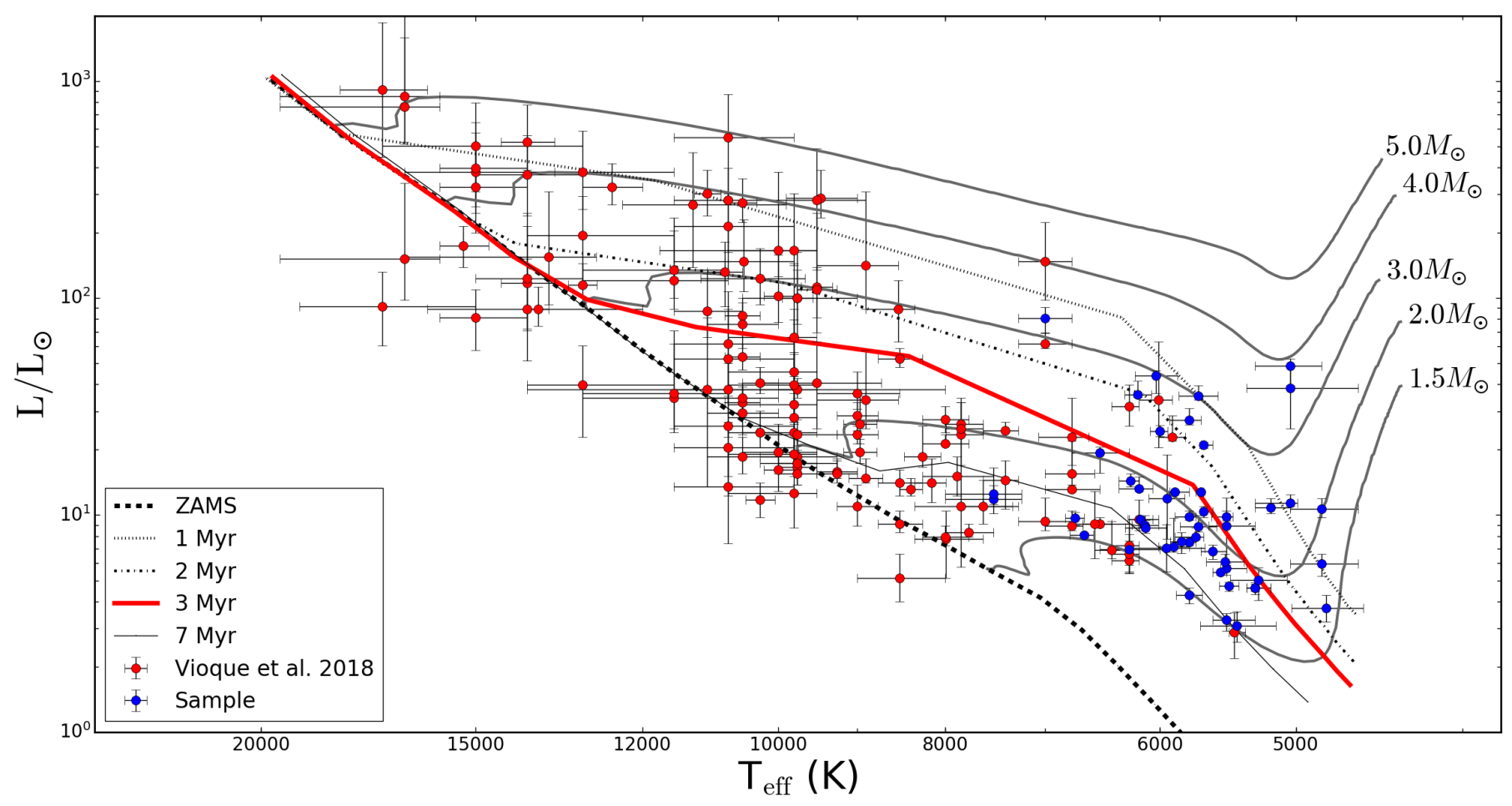

Fig. 1. This HR-diagram shows the IMTT stars sample (blue) and the HAeBe sample from Vioque et al. 2018 (red) that lies within the same mass range as the IMTT stars. The pre-main sequence evolutionary tracks (grey-solid) are from Siess et al. (2000) The isochrones shown are for $1 \mathrm{Myr}$ (dotted), 2 Myr (dash-dotted), 3 Myr (red-solid) and for 7 Myr (black-solid) and the ZAMS (thick-dashed) is defined by the location when the nuclear luminosity provides $99 \%$ of the total stellar luminosity.

and Taurus-Auriga star forming region. The richness of sources in Orion may be due to a high abundance of these IMTT stars in this type of starfoming region also other star forming regions have ages that imply that intermediate mass PMS have already lost their disks. 10 of the stars have previously been described as T-Tau stars while the remaining have previously been identified as intermediate mass stars (IMTT or HAeBe stars) (see table 2). We present the stellar parameters for these stars as found by the method described in the previous subsections in table 3 .

The majority of the stars have masses $\leq 2 M_{\odot}$, only 4 stars have masses $\geq 3 M_{\odot}$ (figure 2 lower panel) in contrast with the sample of Vioque et al. (2018). This is consistent with the fact that stars of higher mass are rarer, and also that higher mass stars evolve faster into A and B stars and leave our predefined temperature range in the HR-diagram in a shorter time. In addition the higher mass stars in the F-K temperature region may not yet have cleared their envelope and may still be embedded. They therefore fall outside our optical selection criteria and do not show in our sample.

Compared with the Vioque et al. (2018) sample the stellar age in each mass bin is on average younger (figure 2 upper panel). This means that for example if we look at stars with mass between 1.5-2.0 $M_{\odot}$ the average age is lower than the typical age of the Herbig stars. This shows that we indeed have possible candidate progenitors of the Herbig stars in the sample. As expected, the stellar age increases with lower mass, because PMS evolutionary timescales decrease with increasing stellar mass. The median age for the sample is $4 \mathrm{Myr}$.

With the help of the fitted photospheric spectrum the infrared excess can be determined. Infrared, millimeter and submillimeter photometry were collected for this sample from the AllWISE, AKARI, Spitzer and Herschel point source catalogs but also from other publications (see table 4 on details for each source star) to build up the SEDs so that the disk can be analysed. In gas rich disks we expect to see an near infrared excess in the SED in contrast to debris disks where the infrared excess first becomes visible beyond $10 \mu \mathrm{m}$. In the sample we can identify 6 disks where the SED resembles that of a debris disk (HBC 415, Brun 252, Brun 555, Brun 656, Ass Cha T2-21, Ass Cha $\mathrm{T} 2-54)$. Since we are interested in gas rich disks these stars remain in our presented sample of sources but are removed from the disk analysis.

\section{Qualitative disk analysis}

In this section, we examine the SED and use archived low resolution mid-infrared spectra from the Combined Atlas of Sources with Spitzer Infrared Spectra (CASSIS) (Lebouteiller et al. 2011) to perform a comparison with the HAeBe and TTauri population in a qualitative analysis of the disks (see table 4 for CASSIS AOR-key). The spectra of three stars, HD 142666, HD 142527 and HD 144432, have been taken from Juhász et al. (2010). By using the spectral slope between 13 and $30 \mu \mathrm{m}$ we classify the disks into disk geometries and compare the distribution with samples of HAeBe stars. We obtain the strength of the $10 \mu \mathrm{m}$ silicate feature from archived low-resolution Spitzer spectra to examine the state of the silicate grain evolution in the disks with those around HAeBe stars. We inspect the mid-infrared spectra for evidence of PAH emission and compare the detection frequency with the HAeBe and lower mass T-Tauri stars. We perform a comparison between the spectral slope and the near infrared excess in order to examine the inner disk opacity. All values measured in the Spitzer spectra for the sources are available in table 5 . 
Table 1. The final IMTT star sample. References are to spectral class and/or $T_{\text {eff }}$, the stars were $T_{\text {eff }}$ is marked with an 'a' are converted from their literature spectral type to temperature using Pecaut \& Mamajek 2013).

\begin{tabular}{|c|c|c|c|c|c|c|c|c|c|c|}
\hline \multicolumn{2}{|c|}{ Catalogue name } & \multicolumn{3}{|c|}{ Right Ascension } & \multicolumn{3}{|c|}{ Declination } & \multirow[t]{2}{*}{ SpT } & \multirow{2}{*}{$\begin{array}{l}\mathrm{T}_{\mathrm{eff}} \\
{[\mathrm{K}]}\end{array}$} & \multirow{2}{*}{$\begin{array}{l}\text { Ref. to } \\
\text { SpT or } T_{\text {eff }}\end{array}$} \\
\hline Name & 2MASS & hh & $\mathrm{mm}$ & ss & deg & amin & asec & & & \\
\hline BX Ari & $\mathrm{J} 02581122+2030037$ & 2 & 58 & 11.23 & 20 & 30 & 3.15 & $\mathrm{~K} 2$ & $5040^{a}$ & Herczeg \& Hillenbrand \\
\hline HBC 338 & J03254982+3110237 & 3 & 25 & 49.83 & 31 & 10 & 23.84 & G8 & $5490^{a}$ & Maheswar et al. (2003) \\
\hline $\mathrm{LkH} \alpha 330$ & $\mathrm{~J} 03454828+3224118$ & 3 & 45 & 48.28 & 32 & 24 & 11.85 & F7 & $6240^{a}$ & Herczeg \& Hillenbrand (2014) \\
\hline RY Tau & $\mathrm{J} 04215740+2826355$ & 4 & 21 & 57.41 & 28 & 26 & 35.53 & & 5945 & Calvet et al. (2004) \\
\hline T Tau & J04215943+1932063 & 4 & 21 & 59.43 & 19 & 32 & 6.44 & & 5700 & Calvet et al. (2004) \\
\hline UX Tau A & J04300399+1813493 & 4 & 30 & 4 & 18 & 13 & 49.44 & G8 & $5490^{a}$ & Akeson et al. (2019) \\
\hline HQ Tau & $\mathrm{J} 04354733+2250216$ & 4 & 35 & 47.33 & 22 & 50 & 21.64 & K0 & $5280^{a}$ & Nguyen et al. (2012) \\
\hline НBC 415 & $\mathrm{~J} 04355415+2254134$ & 4 & 35 & 54.16 & 22 & 54 & 13.4 & G2 & $5770^{a}$ & Herczeg \& Hillenbrand (2014) \\
\hline SU Aur & $\mathrm{J} 04555938+3034015$ & 4 & 55 & 59.39 & 30 & 34 & 1.5 & G4 & $5680^{a}$ & Herczeg \& Hillenbrand (2014) \\
\hline HD 34700 & $\mathrm{~J} 05194140+0538428$ & 5 & 19 & 41.41 & 5 & 38 & 42.78 & G0 Ive & $5920^{a}$ & Mora et al. (2001) \\
\hline CO Ori & J05273833+1125389 & 5 & 27 & 38.34 & 11 & 25 & 38.92 & & 6030 & Calvet et al. (2004) \\
\hline HD 35929 & J05274279-0819386 & 5 & 27 & 42.79 & -8 & 19 & 38.45 & & 7000 & Farrlamb et al. (2015) \\
\hline PDS 115 & $\mathrm{~J} 05281785+0110061$ & 5 & 28 & 17.85 & 1 & 10 & 6.12 & G2 & $5770^{a}$ & Rojas et al. (2008) \\
\hline GW Ori & J05290838+1152126 & 5 & 29 & 8.39 & 11 & 52 & 12.65 & & 5700 & Villebrun et al.(2019) \\
\hline V1650 Ori & J05291144-0608054 & 5 & 29 & 11.44 & -6 & 8 & 5.4 & & 6160 & 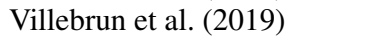 \\
\hline GX Ori & $\mathrm{J} 05300203+1213357$ & 5 & 30 & 2.04 & 12 & 13 & 35.86 & & 5410 & Calvet et al. (2004) \\
\hline RY Ori & J05320993-0249467 & 5 & 32 & 9.94 & -2 & 49 & 46.77 & & 6120 & Villebrun et al.(2019) \\
\hline НBC 442 & J05341416-0536542 & 5 & 34 & 14.16 & -5 & 36 & 54.19 & & 6170 & Manoj et al. (2006) \\
\hline SW Ori & J05341574-0636046 & 5 & 34 & 15.75 & -6 & 36 & 4.68 & G8 & $5490^{a}$ & Hsu et al. (2013) \\
\hline V1044 Ori & J05341646-0536455 & 5 & 34 & 16.46 & -5 & 36 & 45.64 & & 5500 & Villebrun et al. (2019) \\
\hline EZ Ori & J05341856-0504479 & 5 & 34 & 18.57 & -5 & 4 & 47.77 & & 5830 & Calvet et al. $(2004)$ \\
\hline Brun 252 & J05342495-0522055 & 5 & 34 & 24.96 & -5 & 22 & 5.53 & & 5890 & Villebrun et al. (2019) \\
\hline V2149 Ori & J05350519-0514503 & 5 & 35 & 5.21 & -5 & 14 & 50.37 & & 6180 & Villebrun et al. (2019) \\
\hline Brun 555 & J05351511-0444429 & 5 & 35 & 15.13 & -4 & 44 & 42.96 & K2 & $5040^{a}$ & Cohen \& Kuhi (1979) \\
\hline Brun 656 & J05352131-0512126 & 5 & 35 & 21.32 & -5 & 12 & 12.61 & G2 III & $5770^{a}$ & Walker (1969) \\
\hline V815 Ori & J05355263-0505056 & 5 & 35 & 52.63 & -5 & 5 & 5.63 & G7 & $5530^{a}$ & Walker (1969) \\
\hline PR Ori & J05362499-0617324 & 5 & 36 & 24.99 & -6 & 17 & 32.55 & K1 & $5170^{a}$ & Hsu et al. (2013) \\
\hline HD 294260 & & 5 & 36 & 51.27 & -4 & 25 & 39.97 & & 6115 & Calvet et al.(2004) \\
\hline BE Ori & J05370010-0633273 & 5 & 37 & 0.11 & -6 & 33 & 27.33 & G3 & $5720^{a}$ & Hsu et al. $(2012)$ \\
\hline НBC 502 & J05460788-0011568 & 5 & 46 & 7.88 & 0 & -11 & 56.67 & K3 & $4830^{a}$ & Flaherty \& Muzerolle (2008) \\
\hline $\mathrm{LkH} \alpha 310$ & J05471098+0019147 & 5 & 47 & 10.98 & 0 & 19 & 14.77 & G6 & $5590^{a}$ & Fang et al. (2009) \\
\hline HD 288313 A & $\mathrm{J} 05540300+0140217$ & 5 & 54 & 3.01 & 1 & 40 & 21.95 & $\mathrm{~K} 2 \mathrm{~V}$ & $5040^{a}$ & Reipurth et al. (2010) \\
\hline PDS 277 & J08231185-3907015 & 8 & 23 & 11.86 & -39 & 7 & 1.62 & F3 Ve & $6720^{a}$ & Vieira et al. $(2003)$ \\
\hline CR Cha & J10590699-7701404 & 10 & 59 & 6.97 & -77 & 1 & 40.31 & & 4800 & V1llebrun et al.(2019) \\
\hline Ass ChaT2-21 & J11061540-7721567 & 11 & 6 & 15.35 & -77 & 21 & 56.74 & G5 Ve & $5660^{a}$ & Vieira et al. (2003) \\
\hline DI Cha & & 11 & 7 & 20.72 & -77 & 38 & 7.29 & G2 & $5770^{a}$ & Nguyen et al. (2012) \\
\hline CV Cha & $\mathrm{J} 11122772-7644223$ & 11 & 12 & 27.71 & -76 & 44 & 22.3 & K0 & $5280^{a}$ & Manara et al. (2017) \\
\hline Ass ChaT2-54 & J11124268-7722230 & 11 & 12 & 42.67 & -77 & 22 & 22.93 & & 5260 & V1llebrun et al. (2019) \\
\hline HD 135344B & $\mathrm{J} 15154844-3709160$ & 15 & 15 & 48.45 & -37 & 9 & 16.03 & F8 & $6640^{a}$ & Coulson \& Walther (1995) \\
\hline HT Lup & $\mathrm{J} 15451286-3417305$ & 15 & 45 & 12.87 & -34 & 17 & 30.65 & K3 Ve & $4830^{a}$ & Vieira et al. $(2003)$ \\
\hline HD 142666 & $\mathrm{~J} 15564002-2201400$ & 15 & 56 & 40.02 & -22 & 1 & 40.00 & A8 V & $7500^{a}$ & V1eira et al. (2003) \\
\hline HD 142527 & J15564188-4219232 & 15 & 56 & 41.89 & -42 & 19 & 23.25 & & 6500 & Fairlamb et al. (2015) \\
\hline HD 144432 & J16065795-2743094 & 16 & 6 & 57.95 & -27 & 43 & 9.76 & & 7500 & Falrlamb et al. (2015) \\
\hline Haro 1-6 & $\mathrm{J} 16260302-2423360$ & 16 & 26 & 3.03 & -24 & 23 & 36.19 & G1 & $5880^{a}$ & Herczeg \& Hillenbrand (2014) \\
\hline EM*SR 21 & J16271027-2419127 & 16 & 27 & 10.28 & -24 & 19 & 12.62 & & 5950 & Prato et al. (2003) \\
\hline AK Sco & $\mathrm{J} 16544485-3653185$ & 16 & 54 & 44.85 & -36 & 53 & 18.56 & & 6250 & Farrlamb et al.(2015) \\
\hline PDS 156 & $\mathrm{~J} 18272608-0434473$ & 18 & 27 & 26.07 & -4 & 34 & 47.46 & G5 III & $5660^{a}$ & Tisserand et al. (2013) \\
\hline DI Cep & $\mathrm{J} 22561153+5840017$ & 22 & 56 & 11.54 & 58 & 40 & 1.77 & G8 IVe & $5490^{a}$ & Hessman \& Guenther (1997) \\
\hline V395 Cep & $\mathrm{J} 23205208+7414071$ & 23 & 20 & 52.12 & 74 & 14 & 7.08 & & 5470 & Villebrun et al. $(2019)$ \\
\hline
\end{tabular}

${ }^{(a)}$ Temperature derived from spectral type using Pecaut \& Mamajek (2013)

\subsection{Group I vs. Group II}

Spatially resolved observations in scattered light, infrared and submm wavelengths have given rise to the Meeus Group I and Group II classification (Meeus et al.|2001) to represent two different disk geometries (e.g. see Garufi et al. 2018). A similar approach of classifying disks in terms of disk-geometry (full, pre-tansitional and transitional disk) based on their SED, has also been used in T Tauri literature (Luhman \& Mamajek 2012; Esplin et al.2014, Espaillat et al. 2014). Ideally, imaging data is used to establish the geometry of the disks in our sample. Un- fortunately such data are not available for all stars. We can nevertheless use the Group I/Group II classification to infer the disk geometry for our sample. In Appendix $\mathrm{C}$ we verify if our classification in transitional disks (Group I) and self-shadowed/compact (Group II) is supported by published imaging data. We find that for 16 of 18 sources, where spatially resolved image data is available, our classification is supported.

In Group I disks the upper layers of the (flared) disk at spatial scales of tens of AU are directly irradiated by the central star. Almost all Group I disks have a large gap where the dust has been cleared out. That allows the central star to irradiate the outer disk 
Table 2. Table shows the classification as T-Tau star or HAeBe star to confirm the PMS nature of the star and list weather or not this star has previously been referred to as an intermediate mass PMS star. We find 10 new IMTT stars previously classified as T-Tau stars.

\begin{tabular}{|c|c|c|c|}
\hline Name & $\begin{array}{l}\text { Reference to classification } \\
\text { as T-Tau or HAeBe }\end{array}$ & $\begin{array}{l}\text { Known inter- } \\
\text { mediate mass } \\
\text { PMS star }\end{array}$ & $\begin{array}{l}\text { Ref. as inter- } \\
\text { mediate mass } \\
\text { PMS star }\end{array}$ \\
\hline BX Ari & Herczeg \& Hillenbrand $(2014)$ & $\mathrm{Y}$ & Pinzón et al. (2006) \\
\hline НBC 338 & Herbig \& Bell (1988) & $\mathrm{Y}$ & Azimlu et al. (2015) \\
\hline $\mathrm{LkH} \alpha 330$ & Herczeg \& Hillenbrand (2014) & Y & Hein Bertelsen et al. (2016) \\
\hline RY Tau & Calvet et al. (2004) & Y & Calvet et al. (2004) \\
\hline T Tau & Calvet et al. (2004) & Y & Calvet et al. (2004) \\
\hline UX Tau A & Herbst \& Shevchenko (1999) & $\mathrm{Y}$ & Yasui et al. (2019) \\
\hline HQ Tau & Herczeg \& Hillenbrand 2014) & $\mathrm{Y}$ & Pouilly et al. (2020) \\
\hline НBC 415 & Herczeg \& Hillenbrand (2014) & $\mathrm{Y}$ & Yasu1 et al. (2019) \\
\hline SU Aur & Herczeg \& Hillenbrand (2014) & $\mathrm{Y}$ & Calvet et al. (2004) \\
\hline HD 34700 & Sterzik et al. 2005 & Y & Laws et al. $(2020)$ \\
\hline CO Ori & Calvet et al. (2004) & $\mathrm{Y}$ & Calvet et al. (2004) \\
\hline HD 35929 & Vioque et al. (2018) & Y & Vioque et al. (2018) \\
\hline PDS 115 & Rojas et al. (2008) & $\mathrm{N}$ & \\
\hline GW Ori & Calvet et al. (2004) & Y & Calvet et al. (2004) \\
\hline V1650 Ori & Villebrun et al.(2019) & $\mathrm{Y}$ & Villebrun et al. (2019) \\
\hline GX Ori & Calvet et al. $(2004)$ & $\mathrm{Y}$ & Calvet et al. (2004) \\
\hline RY Ori & Villebrun et al. (2019) & $\mathrm{Y}$ & Villebrun et al. (2019) \\
\hline HBC 442 & Villebrun et al. (2019) & $\mathrm{Y}$ & Villebrun et al. (2019) \\
\hline SW Ori & Herbst \& Shevchenko (1999) & $\mathrm{N}$ & \\
\hline V1044 Ori & Calvet et al. (2004) & Y & Calvet et al. 2004) \\
\hline EZ Ori & Calvet et al. (2004) & Y & Calvet et al. (2004) \\
\hline Brun 252 & Villebrun et al. (2019) & Y & Villebrun et al. (2019) \\
\hline V2149 Ori & Villebrun et al. (2019) & $\mathrm{Y}$ & Villebrun et al. (2019) \\
\hline Brun 555 & Kounkel et al. (2017) & $\mathrm{Y}$ & King (1993) \\
\hline Brun 656 & Da Rio et al. (2016) & $\mathrm{N}$ & \\
\hline V815 Ori & Da Rio et al. (2016) & $\mathrm{Y}$ & King (1993) \\
\hline PR Ori & Reipurth et al. (2018) & $\mathrm{N}$ & \\
\hline HD 294260 & Calvet et al. (2004) & Y & Calvet et al. (2004) \\
\hline BE Ori & Da Rio et al. (2016) & $\mathrm{N}$ & \\
\hline HBC 502 & Flaherty \& Muzerolle (2008) & $\mathrm{Y}$ & Matthews et al. (2002) \\
\hline $\mathrm{LkH} \alpha 310$ & Flaherty \& Muzerolle (2008) & $\mathrm{N}$ & \\
\hline HD 288313 A & Reipurth et al. (2010) & $\mathrm{N}$ & \\
\hline PDS 277 & Vioque et al. (2018) & $\mathrm{Y}$ & Vioque et al. (2018) \\
\hline CR Cha & Villebrun et al. (2019) & Y & Villebrun et al. (2019) \\
\hline Ass ChaT2-21 & Herbst \& Shevchenko (1999) & $\mathrm{Y}$ & V1llebrun et al. (2019) \\
\hline DI Cha & Herbst \& Shevchenko (1999) & $\mathrm{Y}$ & Menu et al. (2015) \\
\hline CV Cha & Herbst \& Shevchenko (1999) & $\mathrm{Y}$ & Villebrun et al. (2019) \\
\hline Ass ChaT2-54 & Villebrun et al. (2019) & Y & V1llebrun et al. (2019) \\
\hline HD 135344B & Vioque et al. (2018) & Y & Vioque et al. (2018) \\
\hline HT Lup & Herbst \& Shevchenko (1999) & $\mathrm{N}$ & \\
\hline HD 142666 & Vioque et al. (2018) & Y & Villebrun et al. (2019) \\
\hline HD 142527 & Vioque et al. (2018) & Y & V1oque et al. (2018) \\
\hline HD 144432 & Vioque et al. (2018) & $\mathrm{Y}$ & V1oque et al. (2018) \\
\hline Haro 1-6 & Herczeg \& Hillenbrand (2014) & Y & Menu et al. $(2015)$ \\
\hline EM*SR 21 & Herczeg \& Hillenbrand (2014) & $\mathrm{Y}$ & Menu et al. (2015) \\
\hline AK Sco & Vioque et al. (2018) & $\mathrm{Y}$ & Vioque et al. (2018) \\
\hline PDS 156 & Alfonso-Garzón et al.(2012) & $\mathrm{N}$ & \\
\hline DI Cep & Herbst \& Shevchenko (1999) & $\mathrm{N}$ & \\
\hline V395 Cep & Villebrun et al. (2019) & $\mathrm{Y}$ & Vioque et al. (2018) \\
\hline
\end{tabular}

(Khalafinejad et al.2016). The inner rim of the outer disk scatters stellar radiation and significantly contributes to the SED in the mid- to far-infrared (Honda et al. 2012). In Group II disks the inner rim of the disk cast a shadow on the outer disk (Dullemond \& Dominik 2004). The outer disk, if present, does not receive direct stellar photons and therefore usually shows no or weak emission in scattered light (Garufi et al. 2017), and a 'blue' midto far-IR SED. Other effects that can strongly influence the SED are the disk outer radius (a small outer radius results in a Group II SED, see Dominik et al. 2003), or a misalignment between the inner and the outer disk (Marino et al.2015).
We use the method of Acke et al. (2009) to classify the disks into Group I and Group II objects by based on the infrared slope of the continuum between $13 \mu \mathrm{m}$ and $30 \mu \mathrm{m}$ by using the Spitzer spectra obtained from CASSIS, averaging the flux values in a $0.2 \mu \mathrm{m}$ wide window around $13 \mu \mathrm{m}$ and $30 \mu \mathrm{m}$ separately. The slope of the spectrum is defined as the ratio between the fluxes, $\left[F_{30} / F_{13}\right]$. We define the dividing line between the two groups to be at 2.1 suggested by Khalafinejad et al. (2016) were Group I disks are identified with a flux-ratio $\left[F_{30} / F_{13}\right] \geq 2.1$.

For 7 sources without Spitzer-spectra at 13 and $30 \mu \mathrm{m}$ the classification has instead been based on the photometric infrared 
Table 3. The stellar parameters and disk types for the sample. The effective temperature is found in literature (see references in table 1 ). The distances are Gaia DR2 as derived by Bailer-Jones et al. (2018) unless otherwise indicated. The L and E(B-V) are determined by our SED fitting. Mass and age are determined using Siess et al.(2000) pre-main sequence evolutionary tracks.

\begin{tabular}{|c|c|c|c|c|c|c|c|c|}
\hline Name & 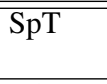 & $\begin{array}{l}T_{\text {eff }} \\
(\mathrm{K})\end{array}$ & $\begin{array}{l}\mathrm{d} \\
{[\mathrm{pc}]}\end{array}$ & $\begin{array}{l}L_{\star} \\
{\left[L_{\odot}\right]} \\
\end{array}$ & $\begin{array}{l}\mathrm{E}(\mathrm{B}-\mathrm{V}) \\
\mathrm{E}(\mathrm{B}-\mathrm{V})\end{array}$ & $\begin{array}{l}M_{\star} \\
{\left[M_{\odot}\right]}\end{array}$ & $\begin{array}{l}\text { Age } \\
\text { [Myr] }\end{array}$ & $\begin{array}{l}\text { Disk } \\
\text { Type }\end{array}$ \\
\hline BX Ari & $\mathrm{K} 2$ & $5040_{-210}^{+240}$ & $238.90_{-3.10}^{+2.97}$ & $48.81_{-9.18}^{+3.90}$ & $4.12_{-0.21}^{+0.01}$ & $3.82_{-0.79}^{+0.05}$ & $0.30_{-0.12}^{+0.17}$ & Ia \\
\hline HBC 338 & G8 & $5490_{-150}^{+40}$ & $290.04_{-6.05}^{+6.32}$ & $5.67_{-0.38}^{+0.22}$ & $0.79_{-0.03}^{+0.01}$ & $1.82_{-0.05}^{+0.18}$ & $4.19_{-1.08}^{+0.72}$ & Ia \\
\hline $\mathrm{LkH} \alpha 330$ & F7 & $6240_{-70}^{+100}$ & $308.43_{-7.37}^{+7.73}$ & $14.39_{-0.92}^{+1.11}$ & $0.98_{-0.03}^{+0.03}$ & $1.93_{-0.07}^{+0.07}$ & $4.66_{-0.26}^{+0.65}$ & Ia \\
\hline RY Tau & & $5945_{-143}^{+143}$ & $133.51_{-30.10}^{+54.81 e}$ & $11.97_{-4.20}^{+6.95}$ & $0.82_{-0.06}^{+0.01}$ & $1.95_{-0.17}^{+0.10}$ & $4.29_{-0.89}^{+1.43}$ & IIa \\
\hline T Tau & & $5700_{-140}^{+140}$ & $143.74_{-1.21}^{+1.22}$ & $8.88_{-0.49}^{+1.09}$ & $0.70_{-0.03}^{+0.05}$ & $1.94_{-0.17}^{+0.21}$ & $4.01_{-1.09}^{+1.35}$ & I \\
\hline UX Tau A & G8 & $5490_{-210}^{+130 a}$ & $139.4 \pm 1.96^{b}$ & $8.91_{-2.88}^{+3.11 b}$ & & $2.34_{-0.43}^{+0.29 b}$ & $1.26_{-0.63}^{+1.03 c}$ & $\mathrm{Ib}$ \\
\hline HQ Tau & K0 & $5280_{-110}^{+60}$ & $158.21_{-5.20}^{+5.56}$ & $4.63_{-0.32}^{+0.27}$ & $1.07_{-0.03}^{+0.02}$ & $1.85_{-0.06}^{+0.05}$ & $3.44_{-0.87}^{+0.62}$ & $\mathrm{IIa}^{j}$ \\
\hline НBC 415 & $\mathrm{G} 2$ & $5770_{-50}^{+110}$ & $165.18_{-1.30}^{+1.31}$ & $7.48_{-0.16}^{+0.40}$ & $0.89_{-0.01}^{+0.02}$ & $1.78_{-0.08}^{+0.07}$ & $5.19_{-0.61}^{+0.96}$ & Debris \\
\hline SU Aur & G4 & $5680_{-20}^{+40}$ & $157.68_{-1.47}^{+1.50}$ & $12.75_{-0.34}^{+0.19}$ & $0.45_{-0.01}^{+0.00}$ & $2.22_{-0.05}^{+0.02}$ & $2.92_{-0.41}^{+0.19}$ & I \\
\hline HD 34700 & G0 Ive & $6060_{-60}^{+80 i}$ & $353.03_{-4.96}^{+6.18}$ & $24.34_{-1.36}^{+1.60}$ & $0.07_{-0.02}^{+0.02}$ & $2.46_{-0.08}^{+0.08}$ & $2.41_{-0.35}^{+0.12}$ & I \\
\hline CO Ori & & $6030_{-170}^{+170}$ & $399.67_{-6.63}^{+6.85}$ & $43.82_{-7.51}^{+2.48}$ & $0.67_{-0.07}^{+0.03}$ & $2.97_{-0.30}^{+0.19}$ & $1.48_{-0.49}^{+0.56}$ & IIa \\
\hline HD 35929 & & $7000_{-250}^{+250}$ & $383.52_{-7.50}^{+7.81}$ & $80.78_{-11.91}^{+9.87}$ & $0.11_{-0.06}^{+0.04}$ & $3.25_{-0.25}^{+0.22}$ & $1.14_{-0.08}^{+0.57}$ & IIa \\
\hline PDS 115 & G2 & $5770_{-100}^{+100}$ & $286.04_{-11.83}^{+12.88}$ & $4.27_{-0.37}^{+0.35}$ & $0.28_{-0.04}^{+0.02}$ & $1.48_{-0.08}^{+0.09}$ & $8.51_{-1.47}^{+1.25}$ & II \\
\hline GW Ori & & $5700_{-150}^{+150}$ & $398.16_{-10.09}^{+10.62}$ & $35.47_{-1.53}^{+4.18}$ & $0.32_{-0.01}^{+0.05}$ & $3.02_{-0.15}^{+0.29}$ & $1.00_{-0.13}^{+0.45}$ & Ia \\
\hline V1650 Ori & & $6160_{-100}^{+100}$ & $342.41_{-5.46}^{+5.63}$ & $9.53_{-0.78}^{+0.25}$ & $0.18_{-0.03}^{+0.01}$ & $1.71_{-0.07}^{+0.05}$ & $6.20_{-0.41}^{+0.96}$ & IIa \\
\hline GX Ori & & $5410_{-275}^{+275}$ & $447.69_{-8.74}^{+9.10}$ & $3.10_{-0.51}^{+0.51}$ & $0.47_{-0.09}^{+0.08}$ & $1.53_{-0.24}^{+0.22}$ & $6.59_{-3.22}^{+5.51}$ & IIa \\
\hline RY Ori & & $6120_{-110}^{+110}$ & $364.79_{-5.11}^{+5.27}$ & $9.01_{-0.28}^{+0.96}$ & $0.57_{-0.02}^{+0.04}$ & $1.69_{-0.05}^{+0.11}$ & $6.65_{-1.34}^{+0.42}$ & IIa \\
\hline HBC 442 & & $6170_{-110}^{+170}$ & $381.67_{-5.64}^{+5.81}$ & $13.20_{-0.28}^{+1.76}$ & $0.13_{-0.00}^{+0.05}$ & $1.90_{-0.06}^{+0.14}$ & $4.75_{-1.14}^{+0.62}$ & IIa \\
\hline SW Ori & G8 & $5490_{-210}^{+100}$ & $375.68_{-4.35}^{+4.45}$ & $3.28_{-0.35}^{+0.26}$ & $0.90_{-0.06}^{+0.04}$ & $1.51_{-0.12}^{+0.17}$ & $7.10_{-2.53}^{+2.30}$ & I \\
\hline V1044 Ori & & $5500_{-140}^{+140}$ & $387.97_{-5.77}^{+5.94}$ & $6.10_{-0.42}^{+0.52}$ & $0.22_{-0.04}^{+0.04}$ & $1.89_{-0.17}^{+0.12}$ & $4.00_{-1.12}^{+1.38}$ & Ia \\
\hline EZ Ori & & $5830_{-87}^{+88}$ & $399_{-23.4}^{+26.5 f}$ & $7.54_{-0.85}^{+0.79}$ & $0.39_{-0.03}^{+0.03}$ & $1.75_{-0.09}^{+0.09}$ & $5.51_{-0.81}^{+1.06}$ & $\mathrm{Ia} / \mathrm{IIa}$ \\
\hline Brun 252 & & $5890_{-120}^{+120}$ & $383.64_{-6.43}^{+6.66}$ & $7.16_{-0.38}^{+2.51}$ & $0.14_{-0.02}^{+0.13}$ & $1.68_{-0.09}^{+0.25}$ & $6.30_{-2.18}^{+1.24}$ & Debris \\
\hline V2149 Ori & & $6180_{-110}^{+110}$ & $388 \pm 5^{d}$ & $35.80_{-0.91}^{+5.61}$ & $0.81_{-0.01}^{+0.06}$ & $2.68_{-0.06}^{+0.22}$ & $2.04_{-0.52}^{+0.07}$ & $\mathrm{Ib} / \mathrm{IIb}$ \\
\hline Brun 555 & $\mathrm{~K} 2$ & $5040_{-50}^{+240}$ & $453.24_{-8.84}^{+9.19}$ & $11.37_{-0.40}^{+1.03}$ & $0.21_{-0.01}^{+0.05}$ & $2.49_{-0.11}^{+0.11}$ & $0.87_{-0.16}^{+0.65}$ & Debris \\
\hline Brun 656 & G2 III & $5770_{-90}^{+150}$ & $466.80_{-9.78}^{+10.19}$ & $27.40_{-1.32}^{+3.62}$ & $0.50_{-0.02}^{+0.05}$ & $2.72_{-0.15}^{+0.21}$ & $1.53_{-0.18}^{+0.48}$ & Debris \\
\hline V815 Ori & G7 & $5530_{-40}^{+60}$ & $397.45_{-4.78}^{+4.86}$ & $5.46_{-0.14}^{+0.09}$ & $0.25_{-0.01}^{+0.00}$ & $1.76_{-0.05}^{+0.06}$ & $4.82_{-0.50}^{+0.45}$ & $\mathrm{Ib}$ \\
\hline PR Ori & K1 & $5170_{-130}^{+110}$ & $408_{-4}^{+4 g}$ & $10.83_{-0.69}^{+1.11}$ & $0.45_{-0.04}^{+0.05}$ & $2.50_{-0.31}^{+0.03}$ & $1.05_{-0.22}^{+0.75}$ & $\mathrm{Ia} / \mathrm{IIa}$ \\
\hline HD 294260 & & $6115_{-168}^{+168}$ & $406.70_{-6.74}^{+6.96}$ & $8.74_{-0.53}^{+0.78}$ & $0.16_{-0.02}^{+0.04}$ & $1.68_{-0.07}^{+0.13}$ & $6.72_{-1.54}^{+0.84}$ & IIa \\
\hline BE Ori & G3 & $5720_{-60}^{+160}$ & $\begin{array}{r}392.27_{-5.29}^{+5.43} \\
\end{array}$ & $7.95_{-0.21}^{+0.80}$ & $1.41_{-0.01}^{+0.05}$ & $1.86_{-0.09}^{+0.10}$ & $4.55_{-0.70}^{+1.13}$ & IIa \\
\hline HBC 502 & K3 & $4830_{-230}^{+210}$ & $410.94_{-8.32}^{+8.66}$ & $10.71_{-0.98}^{+1.26}$ & $1.53_{-0.06}^{+0.07}$ & $2.01_{-0.56}^{+0.52}$ & $0.53_{-0.24}^{+0.46}$ & $\mathrm{Ib}$ \\
\hline $\mathrm{LkH} \alpha 310$ & G6 & $5590_{-100}^{+90}$ & $422.46_{-11.25}^{+11.87}$ & $6.80_{-0.52}^{+0.48}$ & $1.16_{-0.02}^{+0.01}$ & $1.87_{-0.12}^{+0.13}$ & $4.25_{-0.87}^{+0.97}$ & Ia \\
\hline HD 288313 A & $\mathrm{K} 2 \mathrm{~V}$ & $5040_{-440}^{+300}$ & $418_{-17}^{+17 h}$ & $38.47_{-13.20}^{+10.00}$ & $0.34_{-0.21}^{+0.11}$ & $3.47_{-1.59}^{+0.23}$ & $0.52_{-0.39}^{+1.32}$ & - \\
\hline PDS 277 & $\mathrm{~F} 3 \mathrm{Ve}$ & $6720_{-80}^{+90}$ & $342.57_{-4.20}^{+4.31}$ & $9.68_{-0.67}^{+0.61}$ & $0.03_{-0.02}^{+0.02}$ & $1.59_{-0.09}^{+0.05}$ & $8.66_{-1.12}^{+0.33}$ & I \\
\hline CR Cha & & $4800_{-230}^{+230}$ & $186.47_{-0.79}^{+0.81}$ & $3.72_{-0.49}^{+0.57}$ & $0.40_{-0.09}^{+0.10}$ & $1.62_{-0.39}^{+0.28}$ & $1.51_{-0.80}^{+1.59}$ & IIa \\
\hline Ass ChaT2-21 & G5 Ve & $5660_{-70}^{+20}$ & $164.77_{-3.77}^{+3.95}$ & $10.37_{-0.63}^{+0.59}$ & $1.03_{-0.03}^{+0.00}$ & $2.09_{-0.06}^{+0.09}$ & $3.23_{-0.36}^{+0.17}$ & Debris \\
\hline DI Cha & $\mathrm{G} 2$ & $5770_{-50}^{+110}$ & $189.58_{-1.06}^{+1.08}$ & $9.82_{-0.33}^{+0.61}$ & $0.73_{-0.01}^{+0.03}$ & $1.95_{-0.10}^{+0.08}$ & $4.08_{-0.66}^{+0.75}$ & IIa \\
\hline CV Cha & K0 & $5280_{-110}^{+60}$ & $192.17_{-0.97}^{+0.98}$ & $4.63_{-0.15}^{+0.19}$ & $0.46_{-0.02}^{+0.02}$ & $1.85_{-0.05}^{+0.05}$ & $3.44_{-0.87}^{+0.51}$ & IIa \\
\hline Ass ChaT2-54 & & $5260_{-200}^{+200}$ & $202.92_{-16.18}^{+19.19}$ & $5.03_{-0.99}^{+0.71}$ & $0.55_{-0.08}^{+0.03}$ & $1.92_{-0.24}^{+0.08}$ & $3.06_{-1.16}^{+2.24}$ & Debris \\
\hline HD 135344B & $\mathrm{F} 4$ & $6640_{-80}^{+130}$ & $135.26_{-1.40}^{+1.44}$ & $8.09_{-0.24}^{+0.16}$ & $0.21_{-0.01}^{+0.01}$ & $1.50_{-0.01}^{+0.02}$ & $9.07_{-0.25}^{+0.80}$ & $\mathrm{Ib}^{k}$ \\
\hline HT Lup & $\mathrm{K} 3 \mathrm{Ve}$ & $4830_{-230}^{+210}$ & $153.53_{-1.42}^{+1.45}$ & $5.95_{-0.73}^{+0.69}$ & $0.48_{-0.09}^{+0.08}$ & $1.80_{-0.50}^{+0.33}$ & $0.95_{-0.47}^{+0.89}$ & IIa \\
\hline HD 142666 & A8 V & $7500_{-280}^{+500}$ & $147.66_{-1.15}^{+1.17}$ & $11.81_{-1.63}^{+1.93}$ & $0.32_{-0.06}^{+0.05}$ & $1.62_{-0.03}^{+0.13}$ & $8.37_{-1.22}^{+0.83}$ & IIa \\
\hline HD 142527 & & $6500_{-250}^{+250}$ & $156.63_{-1.16}^{+1.18}$ & $19.31_{-3.68}^{+1.29}$ & $0.30_{-0.08}^{+0.03}$ & $2.06_{-0.20}^{+0.18}$ & $3.80_{-1.07}^{+1.80}$ & Ia \\
\hline HD 144432 & & $7500_{-280}^{+500}$ & $154.68_{-1.39}^{+1.40}$ & $12.50_{-1.26}^{+4.06}$ & $0.10_{-0.04}^{+0.09}$ & $1.64_{-0.01}^{+0.19}$ & $8.14_{-1.95}^{+0.63}$ & IIa \\
\hline Haro 1-6 & G1 & $5880_{-110}^{+40}$ & $133.81_{-1.19}^{+1.21}$ & $12.78_{-0.19}^{+0.16}$ & $1.39_{-0.00}^{+0.00}$ & $2.04_{-0.04}^{+0.09}$ & $3.50_{-0.28}^{+0.11}$ & $\mathrm{Ib}$ \\
\hline EM*SR 21 & & $5950_{-300}^{+300}$ & $137.86_{-1.07}^{+1.1}$ & $7.03_{-1.54}^{+2.01}$ & $1.25_{-0.07}^{+0.07}$ & $1.64_{-0.22}^{+0.36}$ & $6.71_{-3.01}^{+3.59}$ & $\mathrm{Ib}$ \\
\hline AK Sco & & $6250_{-250}^{+250}$ & $140.04_{-1.21}^{+1.24}$ & $6.94_{-1.51}^{+0.68}$ & $0.28_{-0.09}^{+0.04}$ & $1.52_{-0.13}^{+0.45}$ & $8.57_{-1.91}^{+3.73}$ & Ia \\
\hline PDS 156 & G5 III & $5660_{-70}^{+20}$ & $397.59_{-6.44}^{+6.64}$ & $21.12_{-0.72}^{+0.50}$ & $1.03_{-0.03}^{+0.00}$ & $2.61_{-0.04}^{+0.08}$ & $1.80_{-0.15}^{+0.07}$ & II \\
\hline DI Cep & G8 IVe & $5490_{-50}^{+110}$ & $430.02_{-5.78}^{+5.94}$ & $9.82_{-0.37}^{+0.63}$ & $0.73_{-0.01}^{+0.03}$ & $2.20_{-0.12}^{+0.09}$ & $2.55_{-0.34}^{+0.63}$ & IIa \\
\hline V395 Cep & & $5470_{-70}^{+70}$ & $188.41_{-1.09}^{+1.11}$ & $4.71_{-0.24}^{+0.20}$ & $0.27_{-0.02}^{+0.02}$ & $1.73_{-0.08}^{+0.09}$ & $4.86_{-0.79}^{+0.9}$ & Ia \\
\hline
\end{tabular}

${ }^{(a)}$ Value from Espaillat et al. 2010 . ${ }^{(b)}$ Value from Akeson et al. 2019. ${ }^{(c)}$ Value from Kraus \& Hilldebrand 2009 . ${ }^{(d)}$ Value from Kounkel et al. 2016. ${ }^{\left({ }^{e}\right)}$ Value from Perryman et al. (1997). (f) Kounkel et al. (2018). ${ }^{(g)}$ Yan et al. (2019) ${ }^{(h)}$ Zucker et al. (2020). ${ }^{(i)} T_{\text {eff }}$ increased see footnote 1 in section 2.5 for explanation. ") $10 \mu \mathrm{m}$ feature shown in Watson et al. (2009) ( ${ }^{(K)}$ Absence of $10 \mu \mathrm{m}$ feature shown in Fedele et al. (2008) 
Table 4. References to photometry used for the SED. The last column contains the AOR-key from the CASSIS database to the Spitzer spectra used for the source. Optical [0.38-1.24 $\mu \mathbf{m}$ ]: 1) Nascimbeni et al.(2016), 2) Henden et al. (2015), 3) Gaia Collaboration et al. (2018), 4) Bailer-Jones (2011), 5) Morel \& Magnenat (1978), 6) Huber et al.(2017), 7) Andruk et al.(2016), 8) Myers et al. (2015), 9)|Vioque et al. (2018), 10)|Manoj et al.(2006), 11) Yuldoshev et al.(2017), 12) Lasker et al.(2008), 13) Hsu et al.(2012), 14) Ammons et al. (2006), 15) Kervella et al. (2019), 16) Hsu et al. (2013), 17) Oelkers et al. (2018), 18) McDonald et al.(2017), 19) Girard et al. (2011), 20) Sartori et al. (2003), 21) Frasca et al. (2018), 22) Kunder et al. (2017). [1.25-2.23 $\mu \mathbf{m}$ ]: 1) Cutri \& et al. (2014), 2) Cutri et al. (2003). [2.24-60 $\mu \mathbf{m}]:$ 1) Cutri \& et al. (2014), 2) Abrahamyan et al. (2015), 3) Ishihara et al. (2010), 4) Evans et al.(2003), 5) Cutri \& et al.|(2012) 6) Esplin et al. (2014), 7) Rebull et al. (2010), 8) Esplin \& Luhman (2019), 9) Megeath et al. (2012), 10) Pillitteri et al. (2013), 11) Getman et al. (2017), 12) Fang et al. (2013), 13) Flaherty \& Muzerolle (2008), 14) Luhman \& Muench (2008), 15) Luhman et al. (2008), 16) Dunham et al.(2015), 17) Merín et al. (2008), 18) 198 (1988), 19) Meng et al. (2017). [61-500 $\mu \mathbf{m}$ ]: 1) Evans et al. (2003), 2) Herschel Point Source Catalogue Working Group et al. (2020), 3) Ribas et al. (2017), 4) Abrahamyan et al. (2015), 5) Wahhaj et al. (2010), 6) Rebull et al. (2010), 7) Sandell et al. (2011), 8) Merín et al. (2008), 9, Benedettini et al. (2018), 10) Dzib et al. (2013), 11) Dunham et al. (2015), 12) Rebollido et al. (2015). [>500 $\mu \mathbf{m}$ ]: 1) Andrews et al. (2013), 2) Mohanty et al. (2013), 3) Dzib et al.(2015), 4) Duchêne (2010), 5) Harris et al.(2012), 6) Galli et al. (2018), 7) Kounkel et al. (2014), 8) Kounkel et al. (2017), 9) Pascucci et al. (2016), 10) Sandell et al. (2011), 11) Ansdell et al. (2018), 12) Cieza et al. (2008), 13) Dzib et al. (2013).

\begin{tabular}{|c|c|c|c|c|c|c|}
\hline Name & $\begin{array}{l}0.38-1.24 \\
\mu \mathrm{m}\end{array}$ & $\begin{array}{l}1.25-2.23 \\
\mu \mathrm{m}\end{array}$ & $\begin{array}{l}2.23-60 \\
\mu \mathrm{m}\end{array}$ & $\begin{array}{l}61-500 \\
\mu \mathrm{m}\end{array}$ & $\begin{array}{l}>500 \\
\mu \mathrm{m}\end{array}$ & $\begin{array}{l}\text { CASSIS } \\
A O R \text {-key }\end{array}$ \\
\hline BX Ari & 1,2 & 1 & $1,2,3$ & & & 14975232 \\
\hline НBC 338 & 1,3 & 1 & $1,2,4$ & 1,2 & & 21868544 \\
\hline $\mathrm{LkH} \alpha 330$ & 1,2 & 1 & $1,2,3,4$ & 1,2 & & 56344816 \\
\hline RY Tau & $1,2,4$ & 2 & $2,3,4,5$ & 3,4 & $1,2,3$ & 26141184 \\
\hline T Tau & $1,2,3,5,6$ & 2 & $1,2,3$ & 2,4 & $1,2,3,4,5,6$ & \\
\hline UX Tau A & $1,2,6,7$ & 2 & $1,2,4$ & $3,4,5$ & $1,2,5$ & 26140928 \\
\hline HQ Tau & 1,6 & 1 & $1,2,3,6$ & 3,6 & 1 & 27057664 \\
\hline НBC 415 & 1,3 & 2 & 1,7 & & 3,6 & 3543040 \\
\hline SU Aur & 1,5 & 2 & $1,2,3,8$ & 2 & 1,2 & 27066880 \\
\hline HD 34700 & $1,2,3,8,9$ & 2 & 2,5 & 4 & & \\
\hline CO Ori & 1,10 & 2 & $1,2,3$ & & & 21870336 \\
\hline HD 35929 & $1,2,3,10,14$ & 2 & $1,2,3$ & & & 10998528 \\
\hline PDS 115 & 1,3 & 2 & $1,2,3$ & & & \\
\hline GW Ori & $1,2,3,5$ & 2 & $1,2,3$ & & & 21870592 \\
\hline V1650 Ori & $1,2,3$ & 2 & $1,2,3$ & & & 21870848 \\
\hline GX Ori & $1,2,3$ & 2 & $1,2,3$ & & & 21871360 \\
\hline RY Ori & $1,2,3$ & 2 & $1,2,3$ & & & 21871616 \\
\hline HBC 442 & $1,2,5,10$ & 2 & $1,2,3,9$ & & & 18832640 \\
\hline SW Ori & 3,11 & 2 & $1,2,3,10$ & 2 & & \\
\hline V1044 Ori & $1,2,3,5$ & 2 & $2,3,9$ & 2 & & 21872640 \\
\hline EZ Ori & $1,2,3,5$ & 2 & $1,2,3,9$ & 2 & & 21872896 \\
\hline Brun 252 & $1,2,3,14$ & 2 & 1 & & & \\
\hline V2149 Ori & $1,2,3$ & 1 & 1,9 & & 7 & 18802176 \\
\hline Brun 555 & $1,2,3,5$ & 2 & 1,11 & & 7,8 & 21874176 \\
\hline Brun 656 & $1,2,3,5$ & 2 & 1,11 & & 7,8 & 21875456 \\
\hline V815 Ori & $1,2,3,5,11$ & 2 & 1,11 & & & 21877504 \\
\hline PR Ori & $1,2,3,16$ & 2 & 1,12 & 2 & 7,8 & 18806784 \\
\hline HD 294260 & $1,2,3$ & 2 & 1,9 & 2 & & 21878528 \\
\hline BE Ori & 2,3 & 2 & $1,2,3,12$ & 2 & & 18815232 \\
\hline HBC 502 & 3 & 2 & $1,11,13$ & 2 & & 12642048 \\
\hline $\mathrm{LkH} \alpha 310$ & 3 & 2 & 1,9 & & & 18756608 \\
\hline HD $288313 \mathrm{~A}$ & $1,2,3,17$ & 1 & 1, & 2 & 7 & \\
\hline PDS 277 & $1,2,3,12,18$ & 1 & 1,2 & 2 & & \\
\hline CR Cha & $1,2,3,19$ & 2 & $1,2,3,14$ & 2 & 9 & 26143744 \\
\hline Ass ChaT2-21 & $1,2,3,19$ & 1 & $1,2,3,15$ & & & 12696320 \\
\hline DI Cha & $1,2,3,14,19$ & 2 & $1,2,3,16$ & 2,11 & 9 & 12697345 \\
\hline CV Cha & $1,2,3,4$ & 2 & $1,2,16$ & 3,11 & & 12697088 \\
\hline Ass ChaT2-54 & $1,2,3,19$ & 2 & 1,16 & 2,4 & & 12695552 \\
\hline HD 135344B & $1,3,19$ & 2 & $1,2,3$ & $1,2,7$ & 10 & 56557088 \\
\hline HT Lup & $1,2,3,4,19$ & 2 & $1,3,17$ & 8,9 & 11 & 22806016 \\
\hline HD 142666 & $1,3,6,10,12,19$ & 2 & $1,2,3$ & 2,4 & & Juhász et al. (2010) \\
\hline HD 142527 & $3,4,10,19,20$ & 2 & 1,18 & 2 & & Juhász et al. \\
\hline HD 144432 & $1,3,10,20$ & 2 & $1,2,3$ & 4 & & Juhász et al. $(\overline{2010})$ \\
\hline Haro 1-6 & $2,3,6$ & 2 & $1,3,19$ & $10,11,12$ & 12,13 & 12698368 \\
\hline EM*SR 21 & $2,3,6$ & 2 & $1,2,3$ & 1,3 & 2 & 12698880 \\
\hline AK Sco & $1,4,9,10,19$ & 2 & $1,2,3,20$ & & & 12700160 \\
\hline PDS 156 & $1,2,3$ & 2 & 1,2 & & & \\
\hline DI Cep & $1,2,3$ & 2 & $1,2,3$ & & & 21887232 \\
\hline V395 Cep & $2,3,21$ & 2 & $1,2,3$ & 4 & & 21887744 \\
\hline
\end{tabular}

Article number, page 8 of 25 

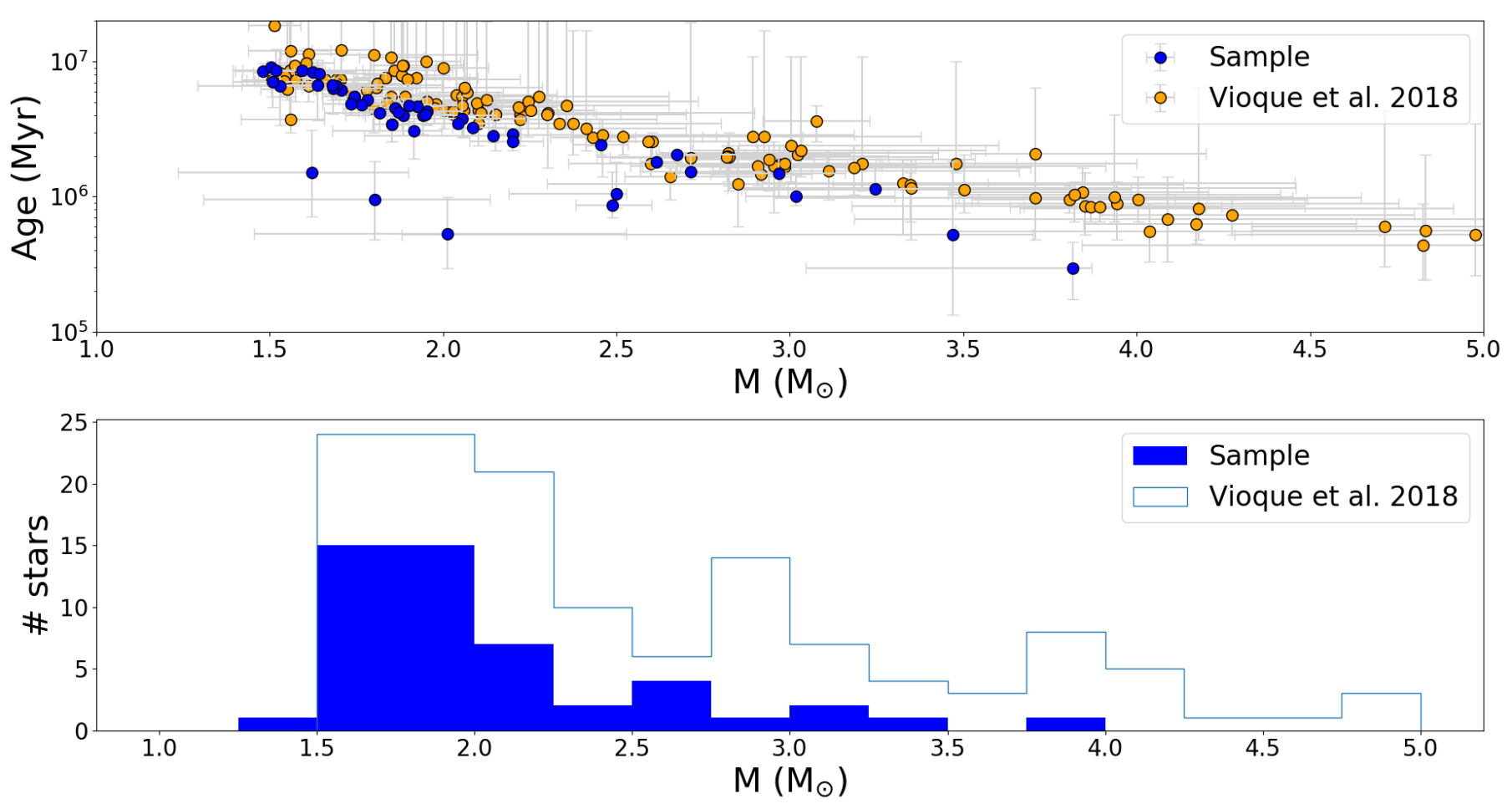

Fig. 2. Comparison between the sample of IMTT stars in this paper with the HAeBe stars sample from Vioque et al. (2018). Only stars up to $5 M_{\odot}$ are shown for the $\mathrm{HAeBe}$ stars in these panels. Upper panel shows the age-mass relation among the IMTT star sample in comparison with the Herbig star sample, the IMTT star sample is with each mass bin on average younger than the HAeBe stars. Lower panel shows the mass distribution in the IMTT star sample compared to the mass distribution among the HAeBe stars.

excess at $60 \mu m, E_{60}$, Acke et al. (2009) suggested that for $\mathrm{HAeBe}$ stars there is a strong correlation between the disk geometries as determined by the $60 \mu \mathrm{m}$ excess and the 13 to $30 \mu \mathrm{m}$ spectral slope. Therefore we make use of a method used by Acke $\&$ van den Ancker (2006) where they for HAeBe stars defined a Group I source having an excess of $E_{60} \geq 10 \mathrm{mag}$. However this limit was set for stars of typical $T_{\text {eff }}$ of $\sim 8000-10000 \mathrm{~K}$. The stars in our sample are cooler (and therefore redder). Since this leads to less excess (see also appendix A) the 10 magnitude excess limit set for the Herbig stars needs to be adjusted. Using BC for PMS stars produced by Pecaut \& Mamajek (2013) we see that there is a difference in bolometric correction of $B C_{J} \sim 0.75$ between HAeBE and IMTTS stars. We therefore use the excess limit $E_{60} \geq 9.25$ mag for Group I disks around the cooler IMTT stars. We measured the flux above the photosphere at $60 \mu \mathrm{m}$ and used the fitted model spectrum to calculate the excess expressed in magnitudes.

We find that 20 sources fall into the Group I category, 19 sources in the Group II category. In addition we have 3 stars that fall in between Group I and Group II due to the uncertainty in their spectral index and 1 source, HD 288313A, where the lack of data makes it impossible using any of these direct methods to determine the Group membership. This result can be compared to (Juhász et al. 2010) where 53 HAeBe stars were categorized into the two Meeus groups using the classification of (van Boekel et al. 2005). In comparison, the HAeBe disk distribution between Group I and Group II are 20 and 33, respectively.

\subsection{Inner disk and disk flaring}

Acke et al. (2009) discovered a correlation between the excess at $7 \mu \mathrm{m}$ and the spectral slope between 13 and $30 \mu \mathrm{m}$. This re- lationship was found for both Group I and Group II disks. The correlation was interpreted as a result of an inner disk casting a shadow on the outer disk. A larger near infrared excess implies a higher inner scale height, resulting in a more shadowed disk with a 'bluer' spectral slope. We wish to investigate if such a correlation is also present in the IMTT star sample.

We therefore measure the excess at $7 \mu \mathrm{m}$ by subtracting the fitted Kurucz model flux from the observed SED at $7 \mu \mathrm{m}$ and then calculate the difference expressed in magnitudes. The results are compared with the HAeBe stars from Acke et al. (2009) and can be seen in Fig 3 (left panel).

The IMTT stars occupy a similar region in figure 3 (left panel). The trend that stars with a larger near infrared excess have a lower $\left[F_{30} / F_{13.5}\right]$ index also holds for the IMTT stars. There is one difference: the maximum $7 \mu \mathrm{m}$ excess is $4.5 \mathrm{mag}$ for IMTT stars and 6 mag for HAeBe stars. In appendix A we show that this difference can simply be explained by the changes in luminosity and temperature of an evolving intermediate mass PMS star, without the need for differences in inner disk structure.

The correlation between the $\left[F_{30} / F_{13.5}\right]$ and $\left[F_{13.5} / F_{7}\right]$ flux ratios described by Acke et al. (2009) for the Group II disks in HAeBe stars is also present among the IMTT stars (figure 3 . right panel). The Group II disks are concentrated in a similar fashion in color and there is less of a spread among the Group I disks in the $\left[F_{13.5} / F_{7}\right]$ ratio then for the HAeBe stars. There is one exception which is the Group II disk of V2149 Ori which is redder, with a lower $\left[F_{13.5} / F_{7}\right]$ flux ratio and lies somewhat outside towards lower $7 \mu \mathrm{m}$ excess the rest of the Group II concentration and has been marked in both panels in figure 3 


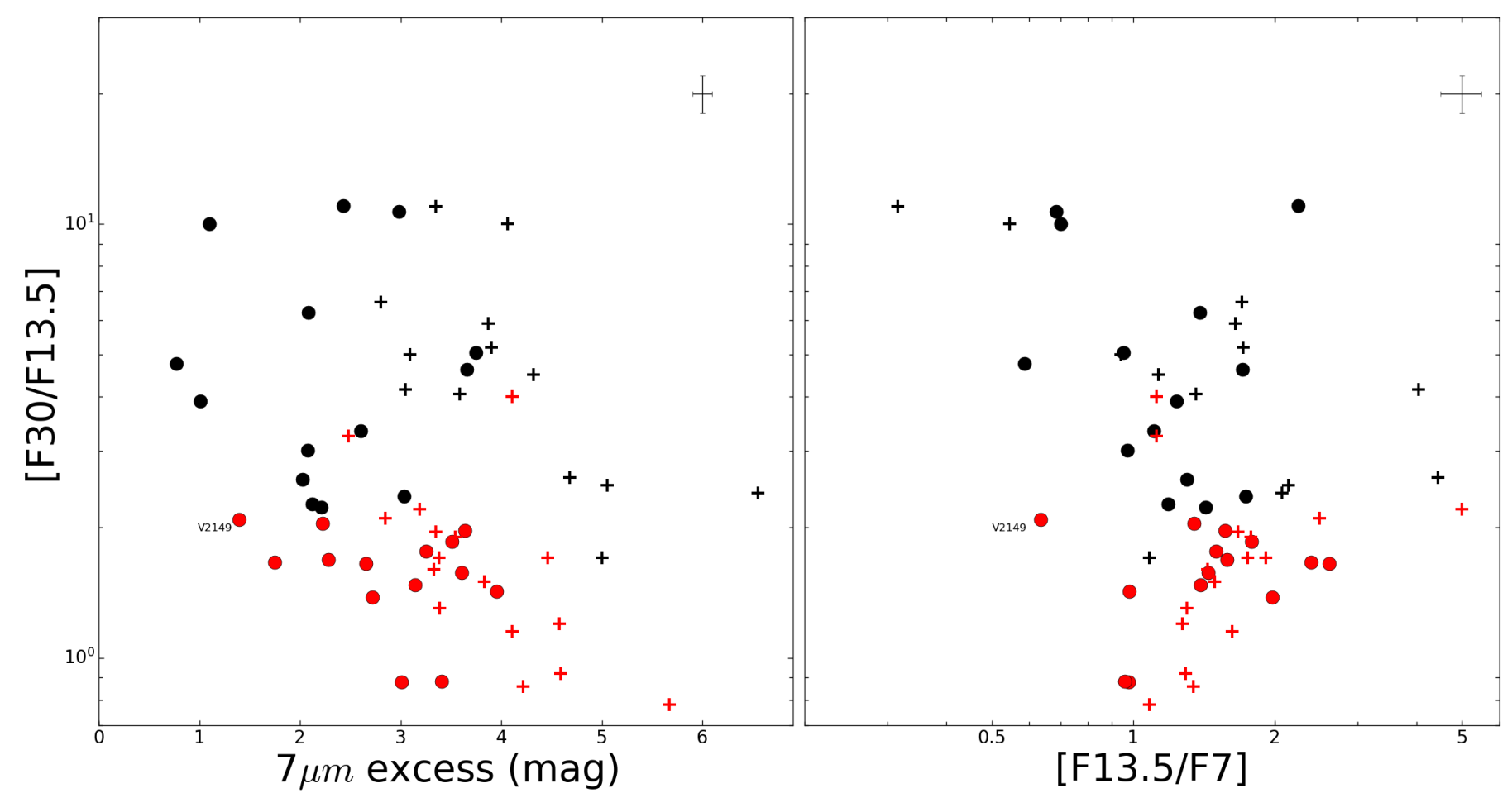

Fig. 3. The crosses are the HAeBe sample from Acke et al. (2009) and the solid circles are the IMTT stars in our sample. Black indicates a Group I source and red a Group II source. The average uncertainty in the measurement is displayed in the top right corner of the panel. Left panel: The relationship between the spectral slope between 13 and $30 \mu \mathrm{m}$ and the $7 \mu \mathrm{m}$ excess. Compared to the HAeBe stars there is a lack of IMTT stars with a $7 \mu \mathrm{m}$ excess above 4 magnitudes. Right panel: The color comparison with the Herbig stars reveal that the distribution is similar to the HAeBe population. The location of V2149 Ori is marked.

\section{3. $10 \mu \mathrm{m}$ silicate feature}

The bulk of dust in protoplanetary disks is made up of silicate grains. The $10 \mu \mathrm{m}$ silicate feature is sensitive to the size of the dust grains, the chemical composition, and the lattice structure in the grains. The shape and strength of the feature can be used as a signpost of dust processing in the disk (van Boekel et al. 2003).

The shape of the silicate emission feature can be measured by the ratio of the flux at $11.3 \mu \mathrm{m}$ and $9.8 \mu \mathrm{m},\left[F_{11.3} / F_{9.8}\right]$ and the peak strength of the feature by the continuum divided flux at $9.8 \mu \mathrm{m}$ see (see van Boekel et al. 2003). As small silicate grains $(0.1 \mu \mathrm{m})$ are removed from the inner disk, primarily by grain growth, the strength of the $10 \mu \mathrm{m}$ band weakens. The evolution also leads to a flatter band shape leading to a higher $\left[F_{11.3} / F_{9.8}\right]$ (van Boekel et al. 2003, Przygodda et al. 2003). The ratio is also sensitive to the presence of a strong crystalline olivine emission band at $11.3 \mu \mathrm{m}$.

To measure the strength and shape of the $10 \mu \mathrm{m}$ silicate feature we first measured the continuum flux at $7 \mu \mathrm{m}$ and at $13.5 \mu \mathrm{m}$ where no prominent emission or absorption bands are present. The underlying continuum was then approximated to be linear between these two points and used to normalize the spectra. We have defined the strength of the $10 \mu \mathrm{m}$ band by measuring the continuum divided flux at $9.8 \mu \mathrm{m}\left(F_{9.8}\right)$ and the shape by the ratio of the continuum divided flux at $11.3 \mu \mathrm{m}$ and at $9.8 \mu \mathrm{m}$ $\left(\mathrm{F}_{11.3} / \mathrm{F}_{9.8}\right)$. We set the threshold for a detectable silicate feature to a peak over continuum strength of 1.2 to clearly separate the emission from the noise in the spectra. In Figure 4 we show the relationship between shape of the $10 \mu \mathrm{m}$ silicate feature and the peak strength at $9.8 \mu \mathrm{m}$. In figure 5 and 6 we show the shape of the $10 \mu \mathrm{m}$ silicate feature as a function of rising peak strength for
Group I and Group II sources respectively. The 3 sources that are in the category Group I/II are shown together with the group of which their spectral index, $\left[F_{30} / F_{13.5}\right]$, lies closest. The absence of a $10 \mu \mathrm{m}$ silicate feature means that there are no small grains of the correct temperature for the emission to be formed. This can come from either a gap in the disk where no silicate grains are present, or that the small grains have grown to a size where the emission is not longer present.

Following the HAeBe star classification (Meeus et al. 2001), we call the disks with silicate feature present Ia and IIa and the disks showing no silicate feature Ib and IIb. Using the Spitzer spectra we have available we are able to make this sub categorization for 15 Group I disks and 17 Group II disks. We find 9 Group Ia disks vs. 6 Group Ib disks and 17 Group IIa disks and no group IIb disk. The 3 disks with spectral index that puts them in between Group I and Group II, 2 are Group Ia/IIa disks and one Group Ib/IIb disk. Among the HAeBe stars the distribution is similar with 15 Group Ia vs. 5 Group Ib and 29 Group IIa vs. 4 Group IIb (Juhász et al. 2010).

We find the relationship between the flux ratio $\left[F_{11.3} / F_{9.8}\right]$ and the peak over continuum flux at $9.8 \mu \mathrm{m}$, in Group Ia and Group IIa disks, follows the same relationship as found among HAeBe and T-Tauri stars by other authors (Varga et al. 2018, van Boekel et al. 2003; Bouwman et al. 2008; Przygodda et al. 2003, Furlan et al. 2009). However, the peak-over-continuum strength distribution of the $10 \mu \mathrm{m}$ silicate feature is more similar to the HAeBe stars than the T-Tauri star distribution (see figure 7), where the T-Tauri stars lack the tail towards higher emission band strength visible in both the IMTT stars and HAeBe stars. Such a trend extends over a much wider stellar and sub-stellar mass range (e.g. Pascucci et al. 2009), and is interpreted in terms 
of an increase in the degree of inner disk settling with decreasing stellar mass, extending to the brown dwarf regime.

We also note that the distribution of disks in the $\left[F_{11.3} / F_{9.8}\right]$ flux ratio clusters around two values: one with low peak over continuum intensity at $9.8 \mu \mathrm{m}$ (weak feature) and with $\left[F_{11.3} / F_{9.8}\right]$ flux ratio of around $0.9-1.1$, consistent with larger grains (around $2 \mu \mathrm{m}$ ), and one region with high peak over continuum intensity (strong feature) and low $\left[F_{11.3} / F_{9.8}\right]$ flux ratio (0.6-0.7) consistent with smaller grains (around $0.1 \mu \mathrm{m}$ ). This could be related to changes in the characteristic grain size from sub-micron to a few microns, and the corresponding non-linear change in grain opacity.

Maaskant et al. (2013) show that HAeBe stars with weak or no silicate emission have a $\left[F_{30} / F_{13.5}\right] \gtrsim 5$ (see also Varga et al. 2018). These disks have large cavities, and a corresponding lack of small warm silicate grains that are responsible for the $10 \mu \mathrm{m}$ silicate band. This aspect is also reflected in the IMTT star sample (table 5). The majority of IMTT stars with strong silicate features have $\left[F_{30} / F_{13.5}\right]$ flux ratios well below 5 . We can therefore conclude that the emission from silicate grains around $10 \mu \mathrm{m}$ behaves like in the HAeBe stars, where the small warm silicate grains are much less abundant in the inner disk due to large cavities.

If the hot innermost disk contains small silicate grains, it should still produce a weak silicate band. In two cases, HD 135344B (Varga et al. 2018) and the lower mass T Tauri star T Cha (which hosts a transitional disk, see Olofsson et al. 2013), interferometric observations suggest that such small hot silicate grains are absent. Therefore the lack of silicate emission in some group I disks is likely due to a combination of a large disk gap and a depletion of small grains in the inner disk.

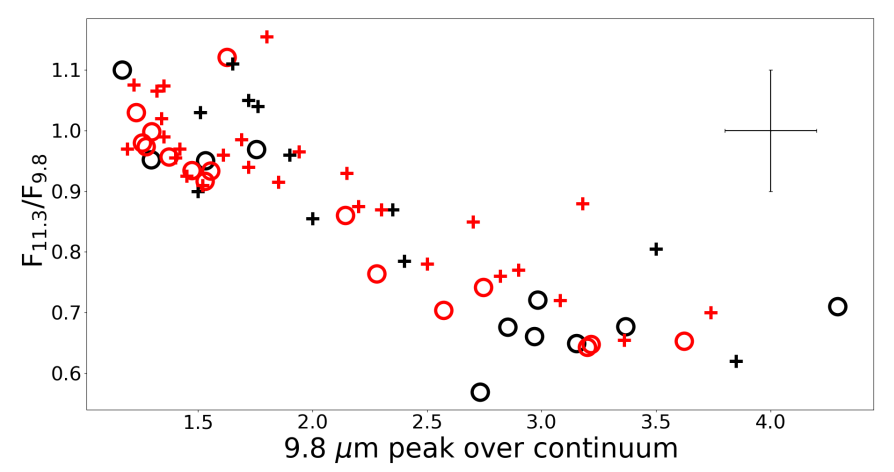

Fig. 4. The degree of processing for the silicate grains in the disks around the IMTT star sample (open circles) and for the HAeBe sample from Juhász et al. (2010) (crosses). Black are Group I disks and red are Group II disks. Smaller grains leads to a high peak over continuum intensity and a low $\left[F_{11.3} / F_{9.8}\right]$ ratio. As grains grow the $\left[F_{11.3} / F_{9.8}\right]$ ratio increases and the peak over continuum intensity weakens.

\subsection{Polycyclic Aromatic Hydrocarbons (PAHs)}

We use low resolution Spitzer spectra between 6 and $13 \mu \mathrm{m}$ to detect the presence of any PAH emission. The PAH emission bands in this wavelength range are at $6.2 \mu \mathrm{m}, 7.7 \mu \mathrm{m}, 8.6 \mu \mathrm{m}$, $11.3 \mu \mathrm{m}$ and $12.7 \mu \mathrm{m}$. We inspect the Spitzer spectra visually and if we see 2 clear PAH bands in the spectra we count this as a detection. In the cases we can only see one clear band we define the PAH detection to be tentative. The result is presented in table 6. The spectral features at $7.7 \mu \mathrm{m}$ and $8.6 \mu \mathrm{m}$ sometimes blend into the blue edge of $10 \mu \mathrm{m}$ silicate features and are therefore often hard to distinguish. The same situation happens on the red edge of the $10 \mu \mathrm{m}$ silicate feature with the $11.3 \mu \mathrm{m}$ PAH band that can even disappear with strong amorphous silicate emission.

We find that the PAH detection rate among the IMTT stars is $27 \%$. Considering also tentative detections the detection rate becomes $44 \%$. In comparison, the detection rate of PAH emission among HAeBe stars is 70\% (Acke et al. 2010) and only $8 \%$ among T-Tauri stars (Geers et al.2006). This positions the IMTT stars, with a higher average luminosity and temperature than the low mass T-Tauri stars but a weaker UV field than the HAeBe stars, in between these two groups in terms of detection rate. The infrared emission from PAH-molecules, for isolated stars, is caused by stellar UV photons that excite electronic states in the PAH molecule, which subsequently de-excites through vibrational emission in stretching and bending mode resonances. The PAH detection rate correlates with the $T_{\text {eff }}$ of the star since the UV radiation field is stronger the hotter the star is (Furlan et al. 2006; Acke et al. 2010).

In order for PAHs to be excited they need be in a region optically thin to UV radiation. This could be in the surface of a flaring disk (ex. HD 97048, Lagage et al. 2006), the inner rim of the disk or in a region where small dust grains are removed while PAHs are still present (e.g. disk gaps or in settled disks, Geers et al. 2007, Woitke et al. 2016). It is therefore interesting to consider the $\mathrm{PAH}$ detection rate and strength as a function of disk geometry. In the $\mathrm{HAeBe}$ sample the PAH detection rates for group Group I and Group II sources are $80 \%$ and $64 \%$, respectively, and PAH emission is generally stronger in Group I disks. Both observations can be understood because Group I sources show large gaps and/or dust depleted inner disk regions where the UV photons can freely propagate. In contrast, in group II disks the PAH emission is likely to come from a decoupling of the gas from the dust where the dust has settled towards the midplane but the gas is still flaring (Acke et al.2010); or the disk is small.

We find that the PAH detection rate in the IMTT Group I and Group II sources are $47 \%$ and $17 \%$ respectively. Adding the tentative rate it becomes $73 \%$ Group I and $29 \%$ Group II. We find that the Group I disks around IMTT stars have on average stronger PAH bands than in Group II disks, as is the case for the HAeBe stars. We conclude that the main difference between HAeBe stars and IMTT is the smaller strength of the PAH bands in the IMTT spectra, but that otherwise both samples show more frequent detection in Group I disks.

Some the most prominent PAH features are seen in SR 21, Haro 1-6 and V2149 Ori. The PAH emission around V2149 Ori, which is classified as a Group I/II disk, is likely to arise from the disk (Kim et al.2013) but due to its location, 9 arcmin away from M42s center just outside the HII region, the radiation exiting the PAH might come from the surrounding hotter stars and not from the binary itself.

The intensity of UV radiation also affects the chemistry of the PAH molecules (Acke et al. 2010). In our sample, we find that the peak of the 6.2 and 7.7 micron PAH bands are often shifted to 6.3 and $7.8 \mu \mathrm{m}$. One explanation for this could be that stronger UV radiation field increases the ratio of aromatic to aliphatic hydrocarbons (Sloan et al.2007, Keller et al. 2008). When the UV radiation field decreases the 6.2 and $7.7 \mu \mathrm{m}$ emission band shifts towards the red. It can be observed that when $T_{\text {eff }}$ decreases these emission bands experience this shift consistent with lower UV flux from the central star. The emission at 6.2 and $7.7 \mu \mathrm{m}$ emission is red-shifted in many of the spectra something that can be seen also in cooler T Tauri stars (Acke et al. 2010). 

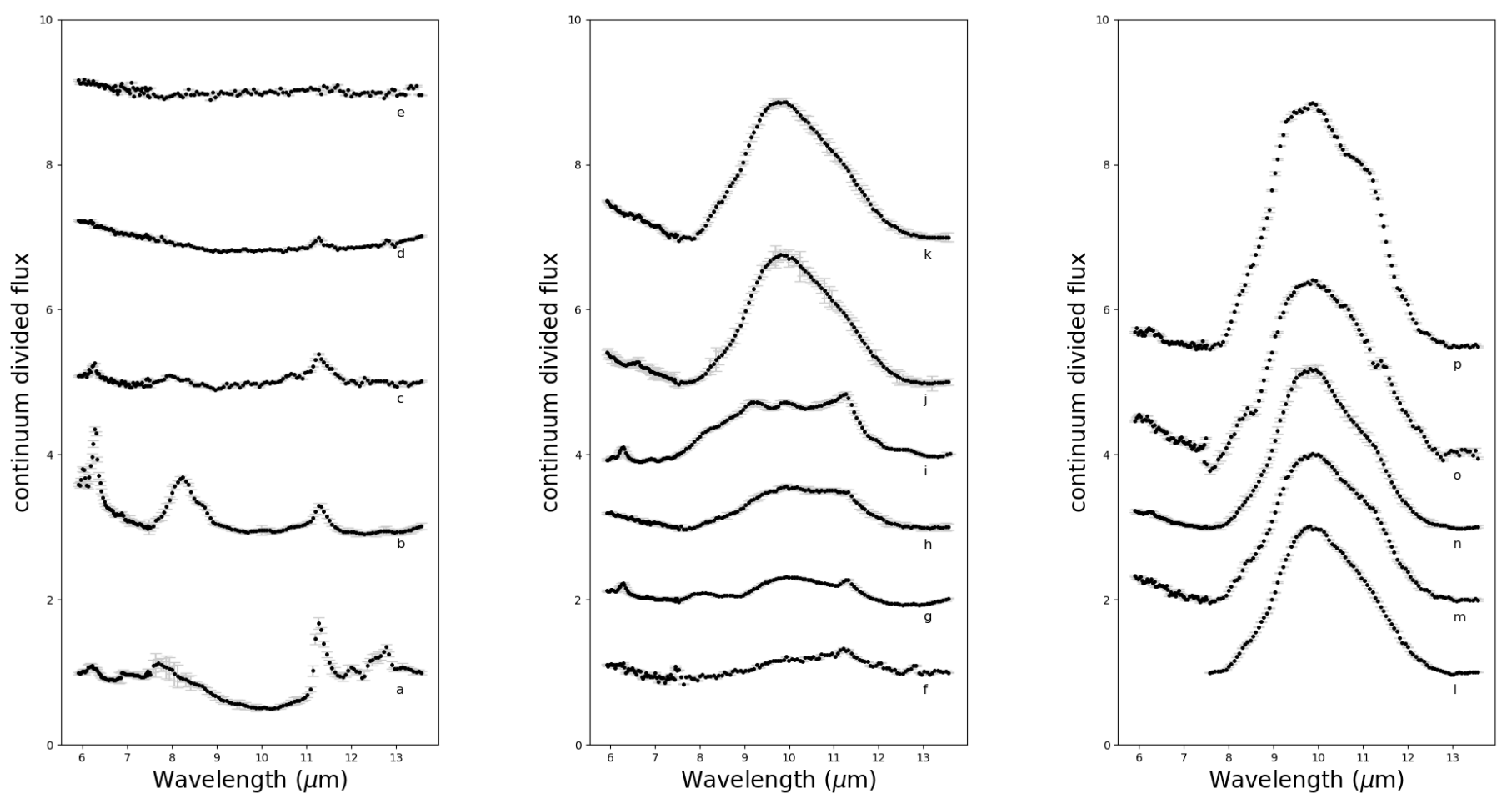

Fig. 5. This shows the continuum divided normalized peak strength over the $10 \mu \mathrm{m}$ feature for those sources classified as Group I. The continuum between 7.5 and $13.5 \mu \mathrm{m}$ has been normalized to 1 . The leftmost panel shows the spectra of sources were no detection of a silicate feature is found: a) Haro 1-6, b) EM*SR 21, c) V815 Ori, d) UX Tau e) HBC 502. From the second left panel the disks with detected silicate feature is shown in ascending order of peak strength: f) HBC 338, g) LkH $\alpha 330$, h) V395 Cep, i) HD 142527, j) GW Ori, k) DI Cep, 1) BX Ari, m) EZ Ori, n) AK Sco, o) $\mathrm{LkH} \alpha 310$, p) V1044 Ori. The scale is indicated in the leftmost panel and is the same for all sources, spectra are shifted to allow comparison.

\section{Discussion}

We have constructed a sample of nearby stars, the IMTT stars, that we consider to be likely progenitors of the class of well studied HAeBe stars. We did so in order to facilitate a better understanding of the evolution of gas-rich protoplanetary disks. We here discuss our findings and try to give them an interpretation.

\subsection{Sample and stellar parameters}

The masses of the IMTT stars found using our method of selection are predominately located in the lower region of the selection mass interval. The median mass of the sample is $1.87 M_{\odot}$. This is partly because of the IMF but mostly because we have created an optically selected sample of the most massive T-Tauri stars. The method only includes stars that have already cleared their stellar envelope which is confirmed by that most stars in the sample show a low to moderate reddening (see table 3). This means we do not have the very youngest and more massive stars in our sample. The lack of higher mass stars $\left(\geq 4 M_{\odot}\right)$ in our specified spectral type range is consistent with a shorter evolutionary timescale for higher mass stars, and the fact that they are less frequent. Within our distance limit of $500 \mathrm{pc}$, we expect very young and massive PMS stars to be present only in the Orion SFR. For stars having a high extinction (for example a high inclination, near edge-on disk or remaining cloud material in a Lada class $0 / \mathrm{I}$ object) our luminosity and mass estimates could be lower than the real values.

The Gaia DR2 parallaxes for the final sample where compared with the new EDR3 release parallaxes. No significant differences where found for stars with RUWE $\leq 1.4$.
To determine the mass and age we use Siess et al. (2000) pre-main sequence stellar evolutionary tracks using a solar like metallicity $(\mathrm{z}=0.01)$. We note that stellar evolution is dependent on metallicity and that in turn masses and ages are affected by the choice of metallicity in the stellar models. A higher metallicity results in higher masses and older stars. However assuming a solar metallicity in the solar neighbourhood corresponds well with G, F and early B-type stars (Sofia \& Meyer 2001; Przybilla et al. 2008).

\subsection{The disks}

We have shown that we find similar disks to the HAeBe sample of Juhász et al. (2010) where the Group I disks are 38\% of the population. Among the IMTT stars there is a more even distribution with $51 \%$ Group I which is not too different from the $\mathrm{HAeBe}$ stars given the sample size. Comparing the SEDs we see that they are similar except for the most extreme $7 \mu \mathrm{m}$ excess cases that can be understood by bolometric correction effects. Comparing the spatially resolved data in literature for the IMTT star sample with that of the HAeBe stars we see that the disks around IMTT stars also show gaps, rings and spirals suggesting disks around IMTT stars are not so very different from those around the HAeBe stars.

$60 \%$ of the Group I disks and all Group II disks show silicate emission at $10 \mu \mathrm{m}$ in our sample. In the HAeBe sample (Juhász et al. 2010) silicate emission is present in $75 \%$ of the Group I disks and $87 \%$ of the Group II disks. The relationship between the strength and shape of the $10 \mu \mathrm{m}$ silicate feature (figure 4 and figure 7 top and middle panel) is consistent with the relationship found among the HAeBe stars (van Boekel et al. 2005) which 

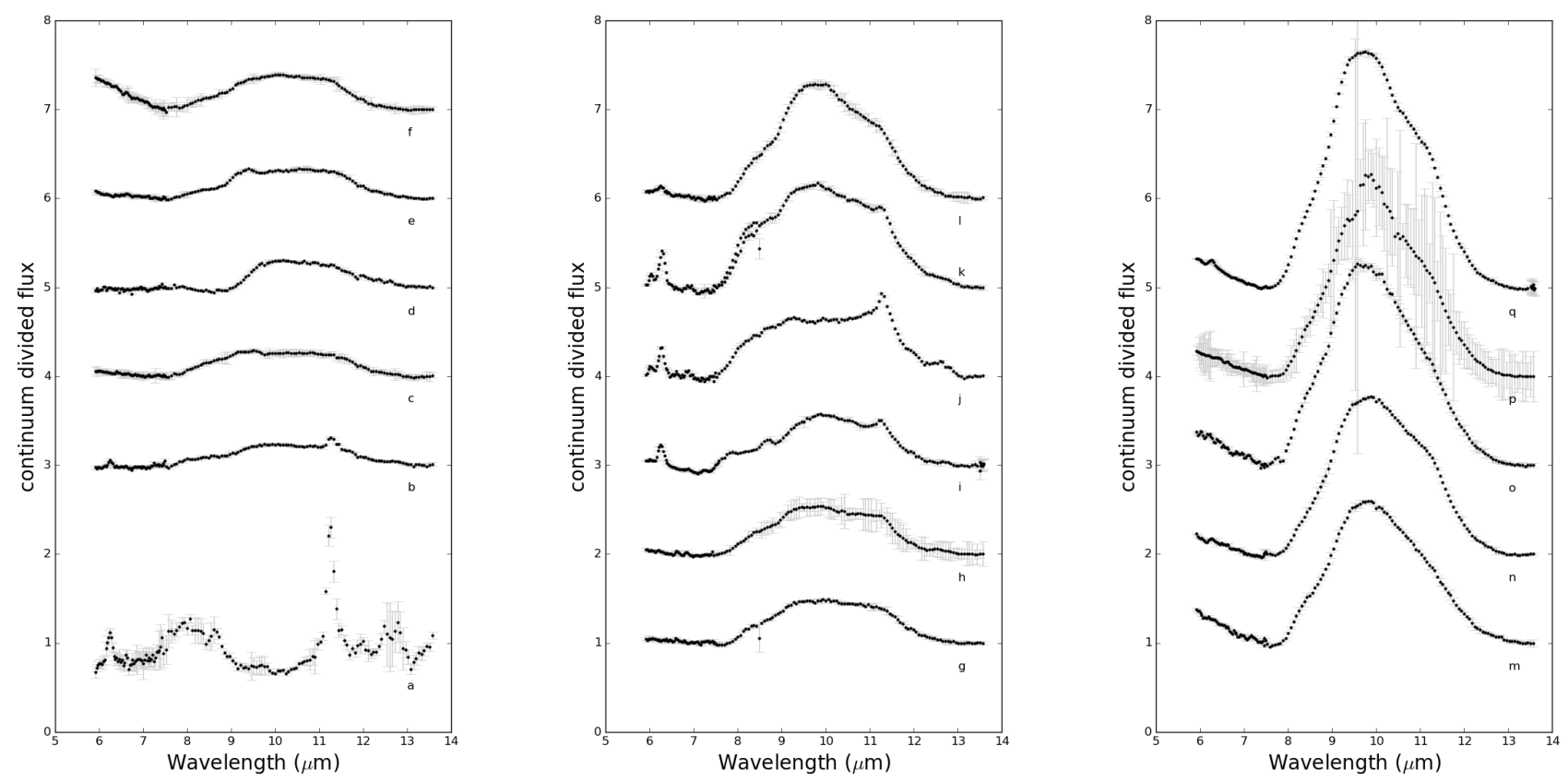

Fig. 6. The continuum divided normalized peak strength over the $10 \mu \mathrm{m}$ feature for those sources classified as Group II. The continuum between 7.5 and $13.5 \mu \mathrm{m}$ has been normalized to 1 . Only a) V2149 Ori, do not have a detectable silicate feature. The other sources then follow in ascending order of peak strength: b) HD 294260, c) CO Ori, d) PR Ori, e) HD 35929, f) DI Cha, g) BE Ori, h) HT Lup, i) HD 142666, j) GX Ori, k) HBC 442 l) V1650 Ori, m) CR Cha, n) CV Cha, o) RY Ori, p) RY Tau, q) HD 144432. The scale is indicated in the leftmost panel and is the same for all sources, spectra are shifted to allow comparison.
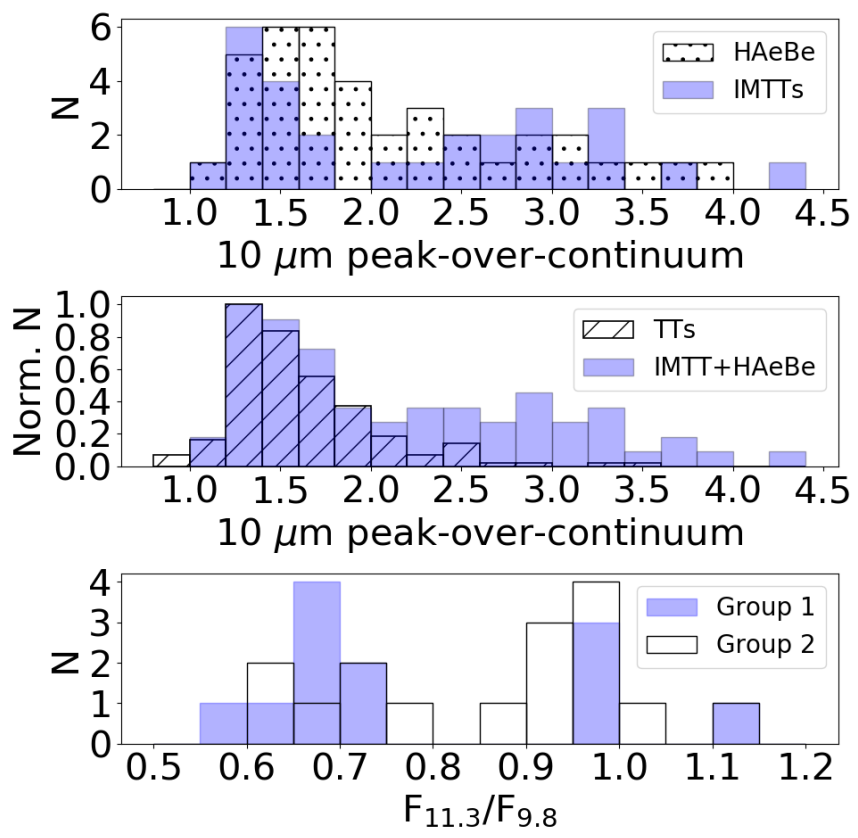

Fig. 7. Upper panel: The $10 \mu \mathrm{m}$ peak-over-continuum distribution among the HAeBe stars Juhász et al. (2010) in comparison with the IMTT star sample. Middle panel: The $10 \mu \mathrm{m}$ peak-over-continuum distribution among T-Tauri stars (Furlan et al. 2011) in comparison with the intermediate mass star samples (IMTTs+HAeBe). Because of the large difference in sample size the distributions have been normalized so that their peak value equals 1 . Lower panel: The distribution in the $\mathrm{F}_{11.3} / \mathrm{F}_{9.8}$ ratio clearly showing the separation between the large grains group and the smaller grains group among the IMTT stars and that Group 1 disks more commonly have smaller grains. suggests that in terms of silicate grain size and growth the grains in IMTT disks are very similar to the HAeBe disks. The emission from silicate dust grains at $10 \mu \mathrm{m}$ in the IMTT sample can be seen in more than half the Group I disks, with a wide silicate strength distribution, from PAH dominated disk without a silicate feature to very strongly peaked features. A silicate emission feature at $10 \mu \mathrm{m}$ is present in all Group II disks (with the exception of V2149 Ori) with on average lower peak strength than in the Group I sources. This may be explained in the following way: The small silicate grains that gives rise to the solid state feature at $10 \mu \mathrm{m}$ are missing in some Group I disks, either because they have grown to a distribution above $\sim 5 \mu \mathrm{m}$ (Olofsson et al. 2013) or because the disk has a cleared-out cavity at the desired temperature and location where the emission is normally coming from (Maaskant et al.2013). In shape and strength the IMTT silicate feature cluster in two groups: one with small pristine grains (strong, peaked feature) and one consistent with more processed grains (weak, broad feature). This can be seen in figure 7 This grouping is not present in the silicate emission feature distribution for the T-Tauri stars (Furlan et al. 2009) nor for the HAeBe stars (Juhász et al. 2010). About twice as many Group I sources show signs of more pristine small silicate grains while for the Group II disks seems to the opposite with more disks having a more processed grain population with larger silicate grains.

The detection rate for emission of PAH-molecules in the sample is $27 \%$. This puts the IMTT stars in between the HAeBe (about 70\%) and the T-Tauri stars (about 8\%). The PAH bands are generally weak with only a few exceptions. This is most likely because IMTT stars are cooler than the HAeBe stars and therefore have a lower UV flux, needed to excite the PAHs. As luminosity and temperature decreases so does the relative contrast of the PAH emission to the continuum and the PAHs disap- 
pear among the classical T-Tauri stars. The relatively weak PAH emission could also be a reason for why we find weaker silicate emission in our sample and why the Group Ib disks are less frequent in the IMTT star sample than among the HAeBe stars, since weaker PAH emission would make any low level silicate emission easier to distinguish.

We find that spatially resolved data available in the literature (see Appendix C) confirms our classification in most cases. Disk features such as spirals and gaps are also present among the IMTT stars suggesting that the disks are already evolved. There are indications that planets form at early stages of disk dissipation and that the process happens quickly (Andrews et al.2018; Long et al. 2018; Zhang et al. 2018; van der Marel et al. 2019) and the transition disk lifetime is thought to be short, $\sim 0.5 \mathrm{Myr}$ (Cieza et al. 2007). Looking at the similarity between IMTT and $\mathrm{HAeBe}$ disks this suggests that disk dissipation and planet formation starts early in some disks and later in others. Considering that disk lifetimes are typically of the order of a few Myr (Alexander et al. 2014; Cieza et al. 2007), it is interesting that the disks around IMTT stars are so similar to the HAeBe stars given that the timescale over which the IMTT star evolves into a HAeBe star is considerably longer.

If there was a evolutionary link between the pre-transitional Group I and Group II disks, one would expect the Group II disks to be the dominant disk type at this earlier stage in the evolution since disk dispersal happens from the inside out in the disk (Koepferl et al. 2013), something that is not apparent from the sample presented here. For these disks to show up in both the HAeBe and IMTT star sample they need to have a significant lifetime.

Disk gaps can form early in the IMTT stars as well as late in the HAeBe stars or maybe even earlier and persist for a long time period. In order to prevent the inner disk in a gapped disk from quickly empty onto the star, it needs to be continuously replenished by the outer disk gas, and some dust, that crosses the gap to the inner disk. If the disk dissipation can be postponed to the later stages of $\mathrm{HAeBe}$ stars, one may expect to find a higher fraction of IMTT stars with gapless disks. But our sample contains roughly equal amounts of Group I (gapped) and Group II (selfshadowed) disks. In addition, the ratio is not very different from that in the HAeBe stars. This may point to a lifetime of gapped disks that is significant. It is hard to imagine a scenario where small Group II disks evolve into large Group I disks. It suggests that Group I and Group II disk evolution are disconnected from each other. Maybe Group I and Group II disks evolve from different parent populations whose parameters are set by the environment at a very early stage.

\section{Conclusions}

The constructed list will serve as a basis for further studies of the IMTT stars and their disks. The sample is not a complete definite list and is likely to grow as more of these stars are identified.

1. With our selection method and criteria we find 49 IMTT stars with infrared excess out of which 6 are debris disks and 44 gas rich disks. Some have been studied earlier as lower mass HAeBe stars and 10 are new IMTT stars previously classified as T-Tauri stars.

2. The sample disks show a more even distribution between Group I and Group II disk geometries than HAeBe samples.

3. The frequency of detected silicate emission is about the same as in the HAeBe samples per disk group and the relationship between strength and shape of the $10 \mu \mathrm{m}$ emission follow the same anti-correlation as the HAeBe stars and points towards grain sizes that are comparable to those of the HAeBe stars.

4. The reddest disks we find $\left[F_{30} / F_{13.5}\right] \geq 5$ are consistent with Group I disks with large central cavities and weak or no silicate emission.

5. The presence of PAH emission is less frequent in the IMTT stars spectra in the sample than among the HAeBe stars but more frequent than among the T-Tauri stars. This is probably caused by an on average decreasing effective temperature of HAeBe stars, IMTT stars and lower mass T Tauri stars, respectively, and the corresponding lower UV flux.

6. Disk dissipation (and therefore planetary formation) seems to takes place early in some disks and not so early in others.

7. Implications are that Group I and Group II disks are two different evolutionary paths a disk can take; and that this is determined at a very early stage of evolution. This is consistent with the conclusion drawn by Garufi et al. (2017).

Acknowledgements. This publication makes use of data products from the Widefield Infrared Survey Explorer, which is a joint project of the University of California, Los Angeles, and the Jet Propulsion Laboratory/California Institute of Technology, funded by the National Aeronautics and Space Administration. This work has made use of data from the European Space Agency (ESA) mission Gaia (https://www. cosmos.esa.int/Gaia), processed by the Gaia Data Processing and Analysis Consortium (DPAC, https://www.cosmos.esa.int/web/ Gaia/dpac/consortium). Funding for the DPAC has been provided by national institutions, in particular the institutions participating in the Gaia Multilateral Agreement. This research has made use of the SIMBAD database, operated at CDS, Strasbourg, France. This research has made use of the VizieR catalogue access tool, CDS, Strasbourg, France (DOI: 10.26093/cds/vizier). The original description of the VizieR service was published in A\&AS 143, 23 We would also like to thank MSc. Erik Gisolf for his contribution to this paper. Finally we thank the anonymous referee for constructive comments that improved the paper significantly.

\section{References}

1988, Infrared Astronomical Satellite (IRAS) Catalogs and Atlases.Volume 7: The Small Scale Structure Catalog., Vol. 7

Abrahamyan, H. V., Mickaelian, A. M., \& Knyazyan, A. V. 2015, Astronomy and Computing, 10, 99

Acke, B., Bouwman, J., Juhász, A., et al. 2010, ApJ, 718, 558

Acke, B., Min, M., van den Ancker, M. E., et al. 2009, A\&A, 502, L17

Acke, B. \& van den Ancker, M. E. 2006, A\&A, 457, 171

Agra-Amboage, V., Dougados, C., Cabrit, S., Garcia, P. J. V., \& Ferruit, P. 2009 , A\&A, 493, 1029

Akeson, R. L., Jensen, E. L. N., Carpenter, J., et al. 2019, ApJ, 872, 158

Akeson, R. L., Walker, C. H., Wood, K., et al. 2005, ApJ, 622, 440

Akiyama, E., Hashimoto, J., Liu, H. B., et al. 2016, AJ, 152, 222

Akiyama, E., Vorobyov, E. I., Baobabu Liu, H., et al. 2019, AJ, 157, 165

Alexander, R., Pascucci, I., Andrews, S., Armitage, P., \& Cieza, L. 2014, in Protostars and Planets VI, ed. H. Beuther, R. S. Klessen, C. P. Dullemond, \& T. Henning, 475

Alfonso-Garzón, J., Domingo, A., Mas-Hesse, J. M., \& Giménez, A. 2012, A\&A, 548, A79

Ammons, S. M., Robinson, S. E., Strader, J., et al. 2006, ApJ, 638, 1004

Andersen, J., Lindgren, H., Hazen, M. L., \& Mayor, M. 1989, The Messenger, 55,45

Andrews, S. M. 2020a, arXiv e-prints, arXiv:2001.05007

Andrews, S. M. 2020b, ARA\&A, 58, 483

Andrews, S. M., Huang, J., Pérez, L. M., et al. 2018, ApJ, 869, L41

Andrews, S. M., Rosenfeld, K. A., Kraus, A. L., \& Wilner, D. J. 2013, ApJ, 771, 129

Andrews, S. M. \& Williams, J. P. 2005, ApJ, 631, 1134

Andrews, S. M., Wilner, D. J., Espaillat, C., et al. 2011, ApJ, 732, 42

Andruk, V. M., Pakuliak, L. K., Golovnia, V. V., et al. 2016, Kinematics and Physics of Celestial Bodies, 32, 260

Ansdell, M., Williams, J. P., Trapman, L., et al. 2018, ApJ, 859, 21

Anthonioz, F., Ménard, F., Pinte, C., et al. 2015, A\&A, 574, A41

Arellano Ferro, A. \& Giridhar, S. 2003, A\&A, 408, L29

Avenhaus, H., Quanz, S. P., Schmid, H. M., et al. 2014, ApJ, 781, 87

Azimlu, M., Martínez-Galarza, J. R., \& Muench, A. A. 2015, AJ, 150, 95

Bailer-Jones, C. A. L. 2011, MNRAS, 411, 435 


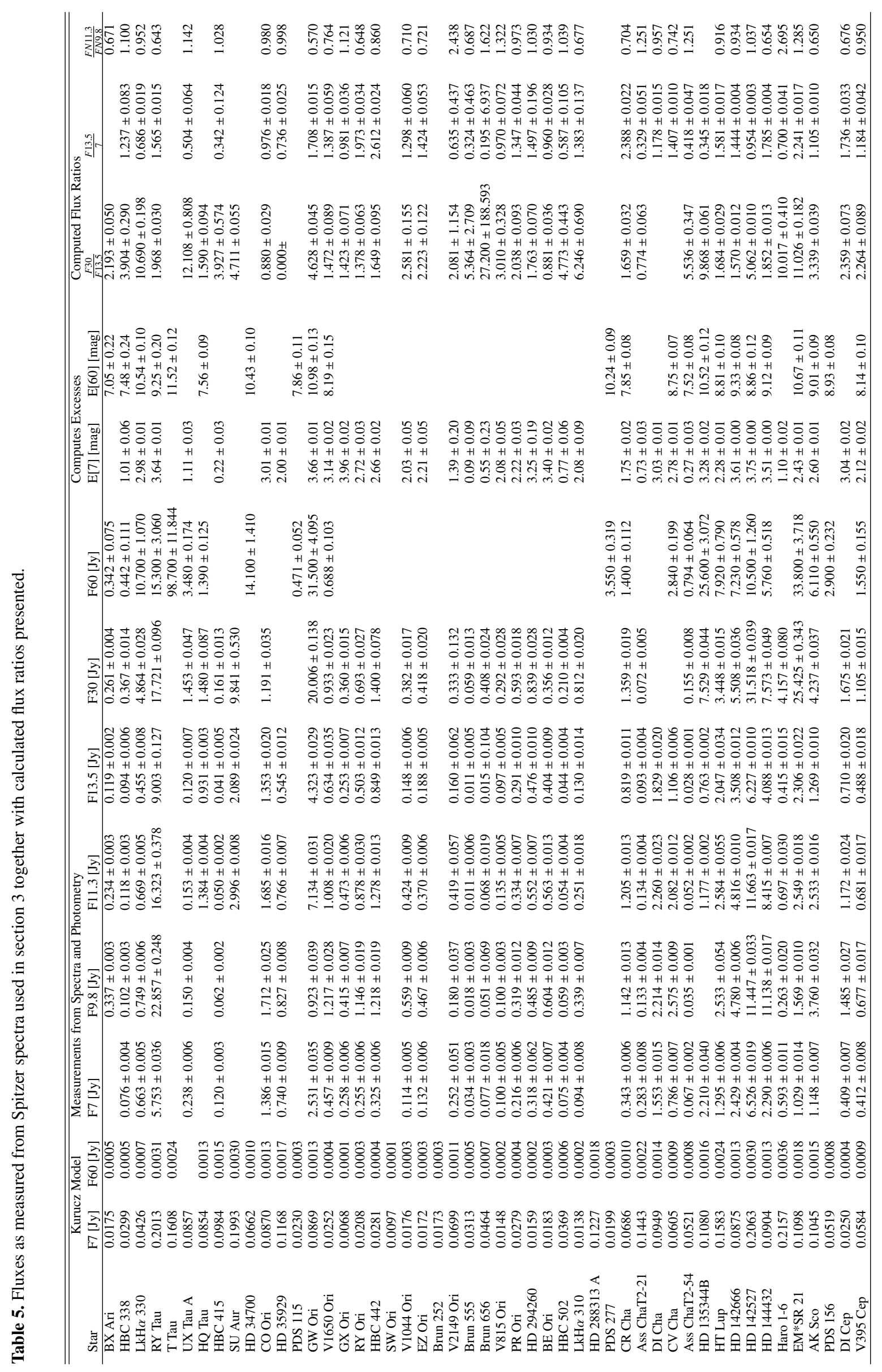


Fig. 8. The SED with fitted Kurucz model. The Kurucz model is in blue, literature photometry is black (see references table 4 ) and Spitzer spectra in red.
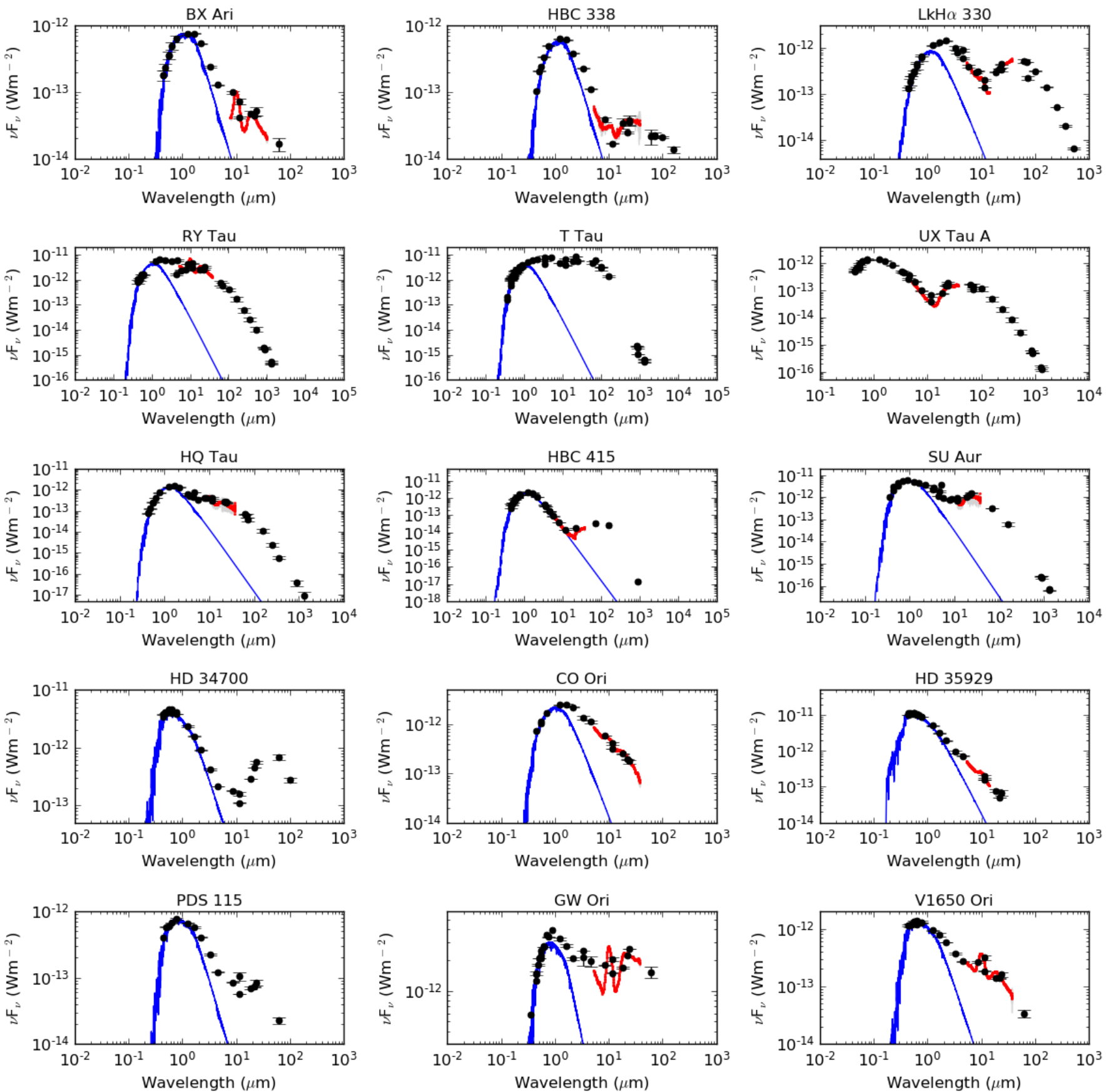

Bailer-Jones, C. A. L., Rybizki, J., Fouesneau, M., Mantelet, G., \& Andrae, R. 2018, AJ, 156, 58

Barsony, M., Koresko, C., \& Matthews, K. 2003, ApJ, 591, 1064

Bastian, U., Finkenzeller, U., Jaschek, C., \& Jaschek, M. 1983, A\&A, 126, 438

Benedettini, M., Pezzuto, S., Schisano, E., et al. 2018, A\&A, 619, A52

Benisty, M., Juhász, A., Facchini, S., et al. 2018, A\&A, 619, A171

Berger, J. P., Monnier, J. D., Millan-Gabet, R., et al. 2011, A\&A, 529, L1

Beuzit, J. L., Vigan, A., Mouillet, D., et al. 2019, A\&A, 631, A155

Bi, J., van der Marel, N., Dong, R., et al. 2020, ApJ, 895, L18

Biller, B., Lacour, S., Juhász, A., et al. 2012, ApJ, 753, L38

Bouwman, J., Henning, T., Hillenbrand, L. A., et al. 2008, ApJ, 683, 479

Brown, J. M., Blake, G. A., Dullemond, C. P., et al. 2007, ApJ, 664, L107

Brown, J. M., Blake, G. A., Qi, C., et al. 2009, ApJ, 704, 496

Calvet, N., Muzerolle, J., Briceño, C., et al. 2004, AJ, 128, 1294

Casassus, S., van der Plas, G. M., Perez, S., et al. 2013, Nature, 493, 191

Cazzoletti, P., van Dishoeck, E. F., Pinilla, P., et al. 2018, A\&A, 619, A161
Cieza, L., Padgett, D. L., Stapelfeldt, K. R., et al. 2007, ApJ, 667, 308

Cieza, L. A., Ruíz-Rodríguez, D., Hales, A., et al. 2019, MNRAS, 482, 698

Cieza, L. A., Swift, J. J., Mathews, G. S., \& Williams, J. P. 2008, ApJ, 686, L115

Cohen, M. \& Kuhi, L. V. 1979, ApJS, 41, 743

Coulson, I. M. \& Walther, D. M. 1995, MNRAS, 274, 977

Csépány, G., van den Ancker, M., Ábrahám, P., et al. 2017, A\&A, 603, A74

Cutri, R. M. \& et al. 2012, VizieR Online Data Catalog, II/311

Cutri, R. M. \& et al. 2014, VizieR Online Data Catalog, II/328

Cutri, R. M., Skrutskie, M. F., van Dyk, S., et al. 2003, VizieR Online Data Catalog, II/246

Czekala, I., Andrews, S. M., Jensen, E. L. N., et al. 2015, ApJ, 806, 154

Da Rio, N., Tan, J. C., Covey, K. R., et al. 2016, ApJ, 818, 59

Daemgen, S., Correia, S., \& Petr-Gotzens, M. G. 2012, A\&A, 540, A46

de Boer, J., Girard, J. H., Canovas, H., et al. 2017, MNRAS, 466, L7

de Leon, J., Takami, M., Karr, J. L., et al. 2015, ApJ, 806, L10

Dominik, C., Dullemond, C. P., Waters, L. B. F. M., \& Walch, S. 2003, A\&A 398,607

Article number, page 16 of 25 

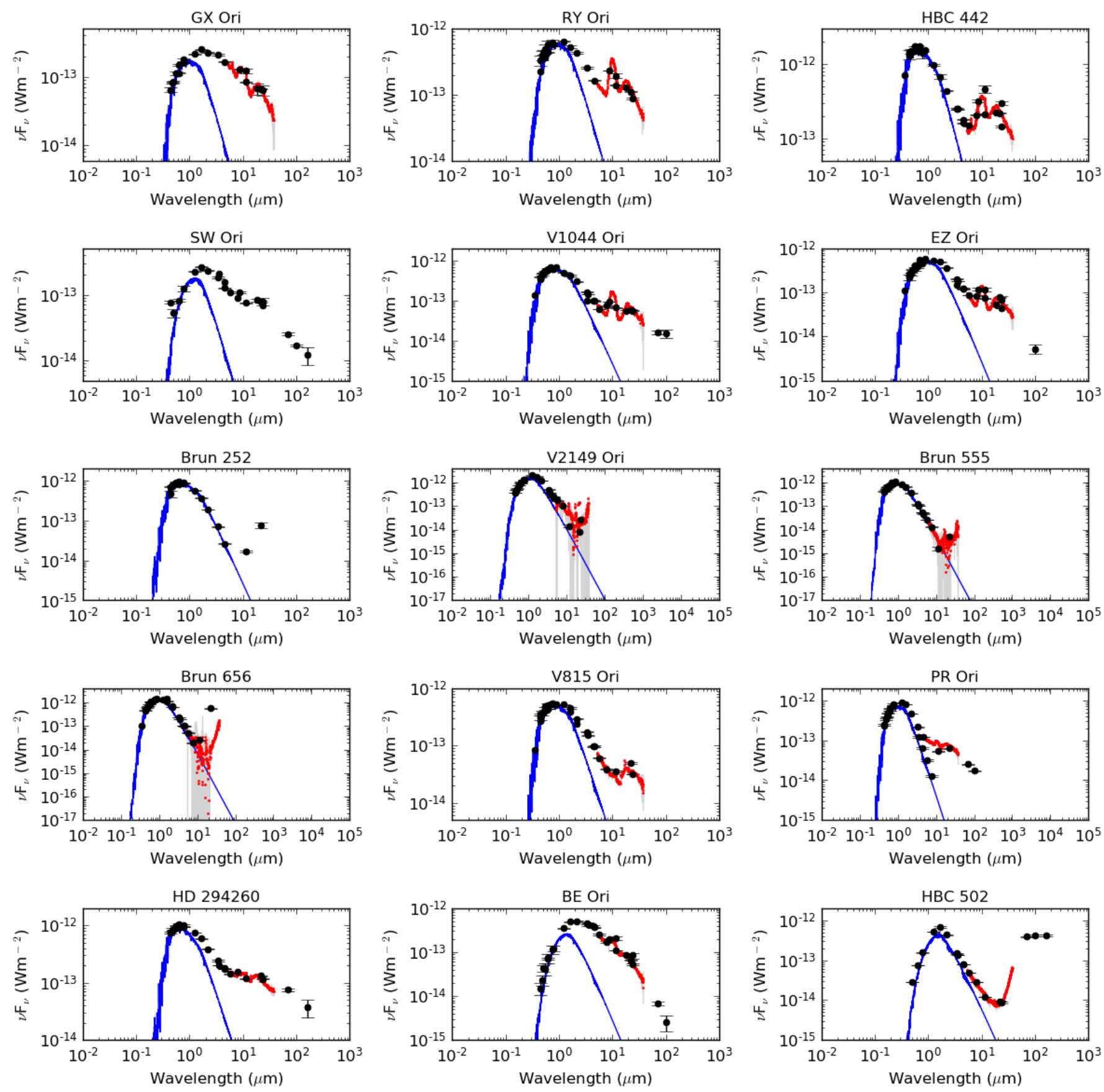

Duchêne, G. 2010, ApJ, 709, L114

Dullemond, C. P. \& Dominik, C. 2004, A\&A, 417, 159

Dunham, M. M., Allen, L. E., Evans, Neal J., I., et al. 2015, ApJS, 220, 11

Dzib, S. A., Loinard, L., Mioduszewski, A. J., et al. 2013, ApJ, 775, 63

Dzib, S. A., Loinard, L., Rodríguez, L. F., et al. 2015, ApJ, 801, 91

Eisner, J. A., Monnier, J. D., Tuthill, P., \& Lacour, S. 2009, ApJ, 698, L169

Espaillat, C., Calvet, N., D’Alessio, P., et al. 2007, ApJ, 670, L135

Espaillat, C., D’Alessio, P., Hernández, J., et al. 2010, ApJ, 717, 441

Espaillat, C., Furlan, E., D'Alessio, P., et al. 2011, ApJ, 728, 49

Espaillat, C., Muzerolle, J., Najita, J., et al. 2014, in Protostars and Planets VI, ed. H. Beuther, R. S. Klessen, C. P. Dullemond, \& T. Henning, 497

Esplin, T. L. \& Luhman, K. L. 2019, AJ, 158, 54

Esplin, T. L., Luhman, K. L., \& Mamajek, E. E. 2014, ApJ, 784, 126

Evans, Neal J., I., Allen, L. E., Blake, G. A., et al. 2003, PASP, 115, 965

Fairlamb, J. R., Oudmaijer, R. D., Mendigutía, I., Ilee, J. D., \& van den Ancker, M. E. 2015, MNRAS, 453, 976

Fang, M., Kim, J. S., van Boekel, R., et al. 2013, ApJS, 207, 5

Fang, M., Sicilia-Aguilar, A., Roccatagliata, V., et al. 2014, A\&A, 570, A118

Fang, M., van Boekel, R., Wang, W., et al. 2009, A\&A, 504, 461
Fedele, D., van den Ancker, M. E., Acke, B., et al. 2008, A\&A, 491, 809 Flaherty, K. M. \& Muzerolle, J. 2008, AJ, 135, 966

Francis, L. \& van der Marel, N. 2020, ApJ, 892, 111

Frasca, A., Guillout, P., Klutsch, A., et al. 2018, A\&A, 612, A96 Fujiwara, H., Honda, M., Kataza, H., et al. 2006, ApJ, 644, L133 Fukagawa, M., Tamura, M., Itoh, Y., et al. 2006, ApJ, 636, L153 Furlan, E., Hartmann, L., Calvet, N., et al. 2006, ApJS, 165, 568 Furlan, E., Luhman, K. L., Espaillat, C., et al. 2011, ApJS, 195, 3 Furlan, E., Watson, D. M., McClure, M. K., et al. 2009, ApJ, 703, 1964

Gaia Collaboration, Brown, A. G. A., Vallenari, A., et al. 2018, A\&A, 616, A1 Galli, P. A. B., Loinard, L., Ortiz-Léon, G. N., et al. 2018, ApJ, 859, 33

Garufi, A., Avenhaus, H., Pérez, S., et al. 2020, A\&A, 633, A82

Garufi, A., Benisty, M., Pinilla, P., et al. 2018, A\&A, 620, A94 Garufi, A., Meeus, G., Benisty, M., et al. 2017, A\&A, 603, A21

Garufi, A., Podio, L., Bacciotti, F., et al. 2019, A\&A, 628, A68

Garufi, A., Quanz, S. P., Avenhaus, H., et al. 2013, A\&A, 560, A105

Geers, V. C., Augereau, J. C., Pontoppidan, K. M., et al. 2006, A\&A, 459, 545

Geers, V. C., Pontoppidan, K. M., van Dishoeck, E. F., et al. 2007, A\&A, 469, L35 

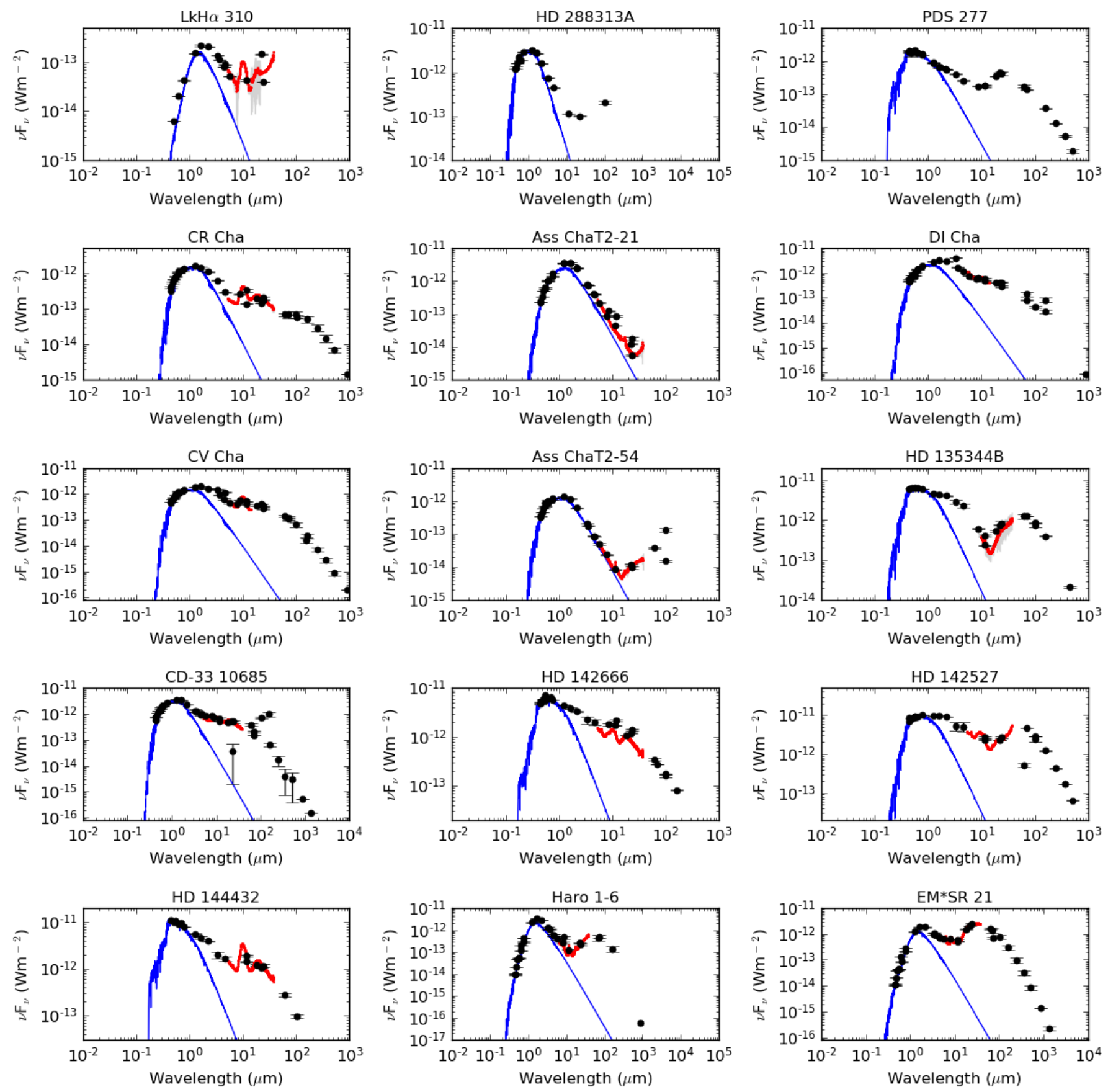

Getman, K. V., Broos, P. S., Kuhn, M. A., et al. 2017, ApJS, 229, 28

Girard, T. M., van Altena, W. F., Zacharias, N., et al. 2011, AJ, 142, 15

Gravity Collaboration, Perraut, K., Labadie, L., et al. 2019, A\&A, 632, A53

Harris, R. J., Andrews, S. M., Wilner, D. J., \& Kraus, A. L. 2012, ApJ, 751, 115

Hartmann, L., Megeath, S. T., Allen, L., et al. 2005, ApJ, 629, 881

Hein Bertelsen, R. P., Kamp, I., van der Plas, G., et al. 2016, A\&A, 590, A98

Henden, A. A., Levine, S., Terrell, D., \& Welch, D. L. 2015, in American Astronomical Society Meeting Abstracts, Vol. 225, American Astronomical Society Meeting Abstracts \#225, 336.16

Henden, A. A., Templeton, M., Terrell, D., et al. 2016, VizieR Online Data Catalog, II/336

Hendler, N., Pascucci, I., Pinilla, P., et al. 2020, ApJ, 895, 126

Henning, T., Pfau, W., Zinnecker, H., \& Prusti, T. 1993, A\&A, 276, 129

Herbig, G. H. 1960, ApJS, 4, 337

Herbig, G. H. 1962, Advances in Astronomy and Astrophysics, 1, 47

Herbig, G. H. \& Bell, K. R. 1988, Third Catalog of Emission-Line Stars of the Orion Population : $3: 1988$

Herbst, W., Herbst, D. K., Grossman, E. J., \& Weinstein, D. 1994, AJ, 108, 1906
Herbst, W. \& Shevchenko, V. S. 1999, AJ, 118, 1043

Herczeg, G. J. \& Hillenbrand, L. A. 2014, ApJ, 786, 97

Herschel Point Source Catalogue Working Group, Marton, G., Calzoletti, L., et al. 2020, VizieR Online Data Catalog, VIII/106

Hessman, F. V. \& Guenther, E. W. 1997, A\&A, 321, 497

Hog, E., Fabricius, C., Makarov, V. V., et al. 2000, VizieR Online Data Catalog, $\mathrm{I} / 259$

Honda, M., Maaskant, K., Okamoto, Y. K., et al. 2012, ApJ, 752, 143

Hsu, W.-H., Hartmann, L., Allen, L., et al. 2012, ApJ, 752, 59

Hsu, W.-H., Hartmann, L., Allen, L., et al. 2013, ApJ, 764, 114

Huang, J., Andrews, S. M., Dullemond, C. P., et al. 2018a, ApJ, 869, L42

Huang, J., Andrews, S. M., Pérez, L. M., et al. 2018b, ApJ, 869, L43

Huber, D., Bryson, S. T., \& et al. 2017, VizieR Online Data Catalog, IV/34

Isella, A., Pérez, L. M., Carpenter, J. M., et al. 2013, ApJ, 775, 30

Ishihara, D., Onaka, T., Kataza, H., et al. 2010, A\&A, 514, A1

Janson, M., Thalmann, C., Boccaletti, A., et al. 2016, ApJ, 816, L1

Jensen, E. L. N., Cohen, D. H., \& Gagné, M. 2009, ApJ, 703, 252

Jensen, E. L. N., Mathieu, R. D., \& Fuller, G. A. 1996, ApJ, 458, 312

Joy, A. H. 1945, ApJ, 102, 168 

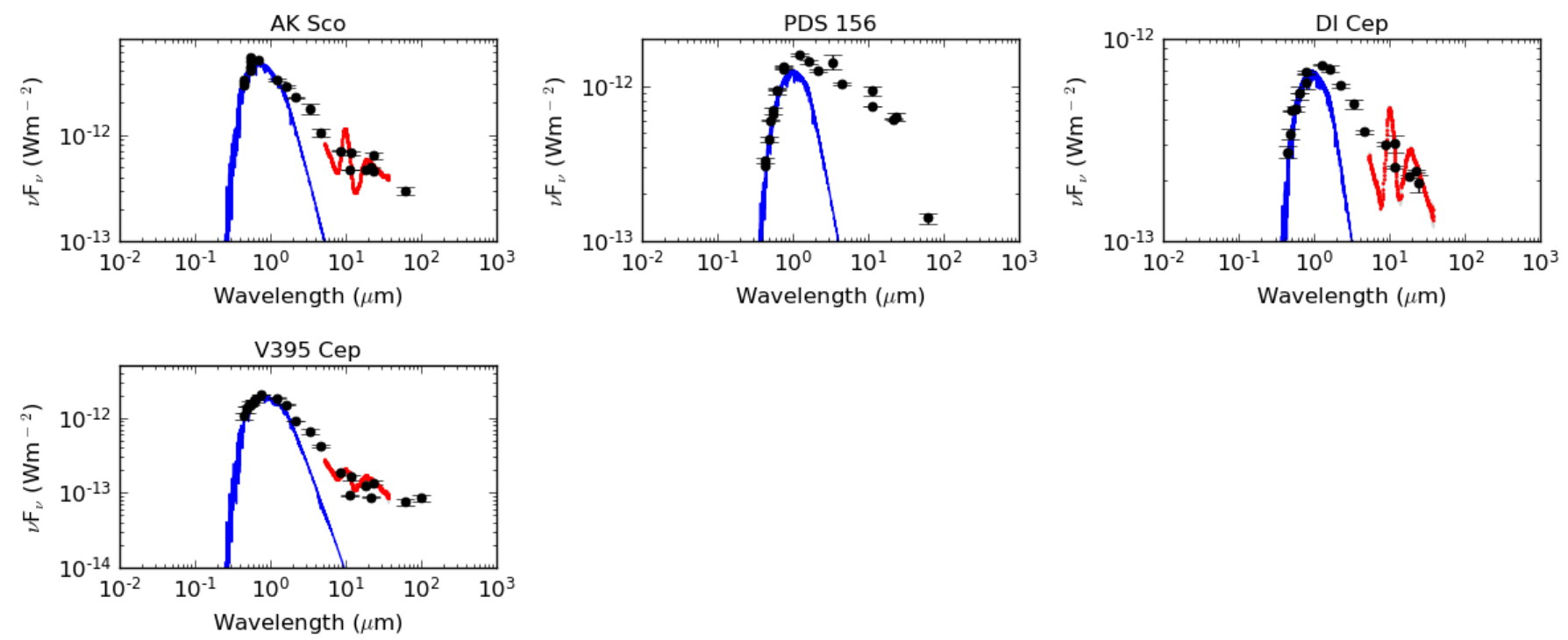

Juhász, A., Bouwman, J., Henning, T., et al. 2010, ApJ, 721, 431

Keller, L. D., Sloan, G. C., Forrest, W. J., et al. 2008, ApJ, 684, 411

Kervella, P., Arenou, F., Mignard, F., \& Thévenin, F. 2019, A\&A, 623, A72

Khalafinejad, S., Maaskant, K. M., Mariñas, N., \& Tielens, A. G. G. M. 2016,

A\&A, 587, A62

Kim, K. H., Watson, D. M., Manoj, P., et al. 2013, ApJ, 769, 149

Kim, S., Takahashi, S., Nomura, H., et al. 2020, ApJ, 888, 72

King, J. R. 1993, AJ, 105, 1087

Koepferl, C. M., Ercolano, B., Dale, J., et al. 2013, MNRAS, 428, 3327

Köhler, R., Petr-Gotzens, M. G., McCaughrean, M. J., et al. 2006, A\&A, 458, 461

Kounkel, M., Covey, K., Suárez, G., et al. 2018, AJ, 156, 84

Kounkel, M., Hartmann, L., Loinard, L., et al. 2014, ApJ, 790, 49

Kounkel, M., Hartmann, L., Loinard, L., et al. 2017, ApJ, 834, 142

Kraus, A. L. \& Hillenbrand, L. A. 2009, ApJ, 704, 531

Kunder, A., Kordopatis, G., Steinmetz, M., et al. 2017, AJ, 153, 75

Kurtovic, N. T., Pérez, L. M., Benisty, M., et al. 2018, ApJ, 869, L44

Labdon, A., Kraus, S., Davies, C. L., et al. 2019, A\&A, 627, A36
Lacour, S., Biller, B., Cheetham, A., et al. 2016, A\&A, 590, A90 Lagage, P.-O., Doucet, C., Pantin, E., et al. 2006, Science, 314, 621 Lasker, B. M., Lattanzi, M. G., McLean, B. J., et al. 2008, AJ, 136, 735 Lavail, A., Kochukhov, O., Hussain, G. A. J., et al. 2017, A\&A, 608, A77 Laws, A. S. E., Harries, T. J., Setterholm, B. R., et al. 2020, ApJ, 888, 7 Lebouteiller, V., Barry, D. J., Spoon, H. W. W., et al. 2011, ApJS, 196, 8 Loinard, L., Torres, R. M., Mioduszewski, A. J., \& Rodríguez, L. F. 2008, in Revista Mexicana de Astronomia y Astrofisica Conference Series, Vol. 34 Revista Mexicana de Astronomia y Astrofisica Conference Series, 14-21

Long, F., Herczeg, G. J., Harsono, D., et al. 2019, ApJ, 882, 49

Long, F., Pinilla, P., Herczeg, G. J., et al. 2018, ApJ, 869, 17

Luhman, K. L., Allen, L. E., Allen, P. R., et al. 2008, ApJ, 675, 1375

Luhman, K. L., Allen, P. R., Espaillat, C., Hartmann, L., \& Calvet, N. 2010, ApJS, 186, 111

Luhman, K. L. \& Mamajek, E. E. 2012, ApJ, 758, 31

Luhman, K. L. \& Muench, A. A. 2008, ApJ, 684, 654

Maaskant, K. M., Honda, M., Waters, L. B. F. M., et al. 2013, A\&A, 555, A64

Mader, S. L., Zealey, W. J., Parker, Q. A., \& Masheder, M. R. W. 1999, MNRAS, 310,331 
Table 6. Index of PAH detections in the Spitzer spectra. The 'x' indicates where a detectable feature is identified.
Ménard, F., Cuello, N., Ginski, C., et al. 2020, A\&A, 639, L1

Meng, H. Y. A., Rieke, G. H., Su, K. Y. L., \& Gáspár, A. 2017, ApJ, 836, 34 Menu, J., van Boekel, R., Henning, T., et al. 2015, A\&A, 581, A107

Merín, B., Jørgensen, J., Spezzi, L., et al. 2008, ApJS, 177, 551

\begin{tabular}{|c|c|c|c|c|c|}
\hline \multirow[t]{2}{*}{ Name } & \multicolumn{5}{|c|}{ PAH emission lines detections } \\
\hline & $6.2 \mu \mathrm{m}$ & $7.7 \mu \mathrm{m}$ & $8.6 \mu \mathrm{m}$ & $11.3 \mu \mathrm{m}$ & $12.7 \mu \mathrm{m}$ \\
\hline \multicolumn{6}{|l|}{ BX Ari } \\
\hline HBC 338 & & & & $\mathrm{x}$ & $\mathrm{x}$ \\
\hline $\mathrm{LkH} \alpha 330$ & $\mathrm{x}$ & & & $\mathrm{x}$ & \\
\hline \multicolumn{6}{|l|}{ RY Tau } \\
\hline \multicolumn{6}{|l|}{$\mathrm{T} \mathrm{Tau}$} \\
\hline UX Tau A & & & & $\mathrm{x}$ & $\mathrm{x}$ \\
\hline \multicolumn{6}{|l|}{ HQ Tau } \\
\hline \multicolumn{6}{|l|}{ HBC 415} \\
\hline \multicolumn{6}{|l|}{ SU Aur } \\
\hline \multicolumn{6}{|l|}{ HD 34700} \\
\hline \multicolumn{6}{|l|}{ CO Ori } \\
\hline \multicolumn{6}{|l|}{ HD 35929} \\
\hline \multicolumn{6}{|l|}{ PDS 115} \\
\hline \multicolumn{6}{|l|}{ GW Ori } \\
\hline V1650 Ori & $\mathrm{x}$ & & & & \\
\hline GX Ori & $\mathrm{x}$ & & $\mathrm{x}$ & $\mathrm{x}$ & $\mathrm{x}$ \\
\hline \multicolumn{6}{|l|}{ RY Ori } \\
\hline HBC 442 & $\mathrm{x}$ & & & $\mathrm{x}$ & \\
\hline \multicolumn{6}{|l|}{ SW Ori } \\
\hline V1044 Ori & $\mathrm{x}$ & & & & \\
\hline \multicolumn{6}{|l|}{ EZ Ori } \\
\hline \multicolumn{6}{|l|}{ Brun 252} \\
\hline V2149 Ori & $\mathrm{x}$ & $\mathrm{x}$ & $\mathrm{x}$ & $\mathrm{x}$ & $\mathrm{x}$ \\
\hline \multicolumn{6}{|l|}{ Brun 555} \\
\hline \multicolumn{6}{|l|}{ Brun 656} \\
\hline V815 Ori & $\mathrm{x}$ & $\mathrm{x}$ & $\mathrm{x}$ & $\mathrm{x}$ & \\
\hline \multicolumn{6}{|l|}{ PR Ori } \\
\hline HD 294260 & $\mathrm{x}$ & & & $\mathrm{x}$ & \\
\hline \multicolumn{6}{|l|}{ BE Ori } \\
\hline HBC 502 & & & & & \\
\hline $\mathrm{LkH} \alpha 310$ & & & & & \\
\hline HD $288313 \mathrm{~A}$ & & & & & \\
\hline PDS 277 & & & & & \\
\hline CR Cha & & & & & \\
\hline Ass ChaT2-21 & & & & $\mathrm{x}$ & \\
\hline DI Cha & & & & & \\
\hline CV Cha & & & & & \\
\hline Ass ChaT2-54 & & & & $\mathrm{x}$ & \\
\hline HD 135344B & & & & $\mathrm{x}$ & \\
\hline HT Lup & & & & & \\
\hline HD 142666 & $\mathrm{x}$ & $\mathrm{x}$ & $\mathrm{x}$ & $\mathrm{x}$ & \\
\hline HD 142527 & $\mathrm{x}$ & & & & \\
\hline HD 144432 & $\mathrm{X}$ & & & & \\
\hline Haro 1-6 & $\mathrm{x}$ & $\mathrm{x}$ & & $\mathrm{x}$ & $\mathrm{x}$ \\
\hline EM*SR 21 & $\mathrm{x}$ & & $\mathrm{x}$ & $\mathrm{x}$ & \\
\hline AK Sco & $\mathrm{X}$ & & & & \\
\hline PDS 156 & & & & & \\
\hline DI Cep & & & & & \\
\hline V395 Cep & & & & & \\
\hline
\end{tabular}

Min, M., Dullemond, C. P., Dominik, C., de Koter, A., \& Hovenier, J. W. 2009, A\&A, 497, 155

Aell, G. F., Johnstone, D., Moriarty-Schieven, G., Fich, M., \& Tothill, N. F. H. 2001, ApJ, 556, 215

Mohanty, S., Greaves, J., Mortlock, D., et al. 2013, ApJ, 773, 168

Monnier, J. D., Harries, T. J., Bae, J., et al. 2019, ApJ, 872, 122

Mora, A., Merín, B., Solano, E., et al. 2001, A\&A, 378, 116

Morel, M. \& Magnenat, P. 1978, A\&AS, 34, 477

Muro-Arena, G. A., Ginski, C., Dominik, C., et al. 2020, A\&A, 636, L4

Muto, T., Grady, C. A., Hashimoto, J., et al. 2012, ApJ, 748, L22

Muzerolle, J., Allen, L. E., Megeath, S. T., Hernández, J., \& Gutermuth, R. A. 2010, ApJ, 708, 1107

Myers, J. R., Sande, C. B., Miller, A. C., Warren, W. H., J., \& Tracewell, D. A. 2015, VizieR Online Data Catalog, V/145

Nascimbeni, V., Piotto, G., Ortolani, S., et al. 2016, MNRAS, 463, 4210

Nguyen, D. C., Brandeker, A., van Kerkwijk, M. H., \& Jayawardhana, R. 2012, ApJ, 745, 119

Oelkers, R. J., Rodriguez, J. E., Stassun, K. G., et al. 2018, AJ, 155, 39

Ohashi, N. 2008, Ap\&SS, 313, 101

Olofsson, J., Benisty, M., Le Bouquin, J. B., et al. 2013, A\&A, 552, A4

Pascucci, I., Apai, D., Luhman, K., et al. 2009, ApJ, 696, 143

Pascucci, I., Testi, L., Herczeg, G. J., et al. 2016, ApJ, 831, 125

Pecaut, M. J. \& Mamajek, E. E. 2013, ApJS, 208, 9

Pérez, L. M., Isella, A., Carpenter, J. M., \& Chandler, C. J. 2014, ApJ, 783, L13

Perryman, M. A. C., Lindegren, L., Kovalevsky, J., et al. 1997, A\&A, 500, 501

Pillitteri, I., Wolk, S. J., Megeath, S. T., et al. 2013, ApJ, 768, 99

Pinilla, P., Benisty, M., Birnstiel, T., et al. 2014, A\&A, 564, A51

Pinilla, P., Tazzari, M., Pascucci, I., et al. 2018, ApJ, 859, 32

Pinzón, G., Seifahrt, A., \& de La Reza, R. 2006, in Revista Mexicana de Astronomia y Astrofisica Conference Series, Vol. 26, Revista Mexicana de Astronomia y Astrofisica Conference Series, 41-42

Pouilly, K., Bouvier, J., Alecian, E., et al. 2020, A\&A, 642, A99

Prato, L., Greene, T. P., \& Simon, M. 2003, ApJ, 584, 853

Przybilla, N., Nieva, M.-F., \& Butler, K. 2008, ApJ, 688, L103

Przygodda, F., van Boekel, R., Àbrahàm, P., et al. 2003, A\&A, 412, L43

Rebollido, I., Merín, B., Ribas, Á., et al. 2015, A\&A, 581, A30

Rebull, L. M., Padgett, D. L., McCabe, C. E., et al. 2010, ApJS, 186, 259

Reipurth, B., Herbig, G., \& Aspin, C. 2010, AJ, 139, 1668

Reipurth, B., Herbig, G. H., Bally, J., et al. 2018, AJ, 156, 25

Reipurth, B. \& Zinnecker, H. 1993, A\&A, 278, 81

Ribas, Á., Espaillat, C. C., Macías, E., et al. 2017, ApJ, 849, 63

Rizzuto, A. C., Dupuy, T. J., Ireland, M. J., \& Kraus, A. L. 2020, ApJ, 889, 175

Rodigas, T. J., Follette, K. B., Weinberger, A., Close, L., \& Hines, D. C. 2014, ApJ, 791, L37

Rojas, G., Gregorio-Hetem, J., \& Hetem, A. 2008, MNRAS, 387, 1335

Rubinstein, A. E., Macías, E., Espaillat, C. C., et al. 2018, ApJ, 860, 7

Sandell, G., Weintraub, D. A., \& Hamidouche, M. 2011, ApJ, 727, 26

Sartori, M. J., Lépine, J. R. D., \& Dias, W. S. 2003, A\&A, 404, 913

Schegerer, A. A., Wolf, S., Hummel, C. A., Quanz, S. P., \& Richichi, A. 2009, A\&A, 502, 367

Schmidt, T. O. B., Vogt, N., Neuhäuser, R., Bedalov, A., \& Roell, T. 2013, A\&A, 557, A 80

Segura-Cox, D. M., Schmiedeke, A., Pineda, J. E., et al. 2020, Nature, 586, 228

Siess, L., Dufour, E., \& Forestini, M. 2000, A\&A, 358, 593

Skinner, S. L., Audard, M., \& Güdel, M. 2016, ApJ, 826, 84

Sloan, G. C., Jura, M., Duley, W. W., et al. 2007, ApJ, 664, 1144

Sofia, U. J. \& Meyer, D. M. 2001, ApJ, 554, L221

Sterzik, M. F., Melo, C. H. F., Tokovinin, A. A., \& van der Bliek, N. 2005, A\&A, 434,671

Stolker, T., Dominik, C., Avenhaus, H., et al. 2016, A\&A, 595, A113

Stolker, T., Sitko, M., Lazareff, B., et al. 2017, ApJ, 849, 143

Takami, M., Karr, J. L., Hashimoto, J., et al. 2013, ApJ, 772, 145

Tanii, R., Itoh, Y., Kudo, T., et al. 2012, PASJ, 64, 124

Tisserand, P., Clayton, G. C., Welch, D. L., et al. 2013, A\&A, 551, A77

Uyama, T., Hashimoto, J., Kuzuhara, M., et al. 2017, AJ, 153, 106

Uyama, T., Hashimoto, J., Muto, T., et al. 2018, AJ, 156, 63

van Boekel, R., Min, M., Waters, L. B. F. M., et al. 2005, A\&A, 437, 189

van Boekel, R., Waters, L. B. F. M., Dominik, C., et al. 2003, A\&A, 400, L21

van der Marel, N., Cazzoletti, P., Pinilla, P., \& Garufi, A. 2016, ApJ, 832, 178

van der Marel, N., Dong, R., di Francesco, J., Williams, J. P., \& Tobin, J. 2019, ApJ, 872, 112

van der Marel, N., van Dishoeck, E. F., Bruderer, S., et al. 2013, Science, 340, 1199

Varga, J., Ábrahám, P., Chen, L., et al. 2018, A\&A, 617, A83

Vieira, S. L. A., Corradi, W. J. B., Alencar, S. H. P., et al. 2003, AJ, 126, 2971

Villebrun, F., Alecian, E., Hussain, G., et al. 2019, A\&A, 622, A72

Megeath, S. T., Gutermuth, R., Muzerolle, J., et al. 2012, AJ, 144, 192 
Vioque, M., Oudmaijer, R. D., Baines, D., Mendigutía, I., \& Pérez-Martínez, R. 2018, A\&A, 620, A128

Voirin, J., Manara, C. F., \& Prusti, T. 2018, A\&A, 610, A64

Wahhaj, Z., Cieza, L., Koerner, D. W., et al. 2010, ApJ, 724, 835

Walker, M. F. 1969, ApJ, 155, 447

Watson, D. M., Leisenring, J. M., Furlan, E., et al. 2009, ApJS, 180, 84

Weaver, W. B. \& Jones, G. 1992, ApJS, 78, 239

Weintraub, D. A. 1990, ApJS, 74, 575

Woitke, P., Kamp, I., Antonellini, S., et al. 2019, PASP, 131, 064301

Woitke, P., Min, M., Pinte, C., et al. 2016, A\&A, 586, A103

Yan, Q.-Z., Zhang, B., Xu, Y., et al. 2019, A\&A, 624, A6

Yasui, C., Hamano, S., Fukue, K., et al. 2019, ApJ, 886, 115

Yuldoshev, Q. X., Ehgamberdiev, S. A., Muminov, M. M., et al. 2017, Kinematics and Physics of Celestial Bodies, 33, 250

Zacharias, N., Finch, C. T., Girard, T. M., et al. 2012, VizieR Online Data Cata$\log , \mathrm{I} / 322 \mathrm{~A}$

Zacharias, N., Monet, D. G., Levine, S. E., et al. 2005, VizieR Online Data Catalog, I/297

Zapata, L. A., Rodríguez, L. F., Fernández-López, M., et al. 2020, ApJ, 896, 132 Zhang, S., Zhu, Z., Huang, J., et al. 2018, ApJ, 869, L47

Zucker, C., Speagle, J. S., Schlafly, E. F., et al. 2020, A\&A, 633, A51 


\section{Appendix A: High RUWE in Final sample}

Here we describe the distance determination for those sources where the Gaia RUWE is larger than 1.4 or where no Gaia parallax was available.

- For LkH $\alpha 330$ we confirm the parallax by using the early Gaia DR3 release and compare the proper motion with stars in a 10 arc minute radius around the star with similar parallaxes. $\mathrm{LkH} \alpha$ 330, located in Perseus, has a distance of 308 pc (Bailer-Jones et al.2018). Examining the DR3 proper motion puts it in a group of 34 stars. The average proper motion of this group is RA 4.19 mas $\mathrm{yr}^{-1}(\sigma=0.37)$ and DEC 6.00 mas $\mathrm{yr}^{-1}(\sigma=0.61)$, compared to the proper motion of

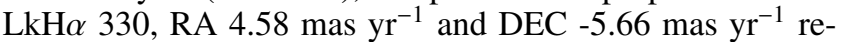
spectively. Within the group the range of parallaxes are 2.96 to 3.99 mas. The DR2 parallax of $\mathrm{LkH} \alpha 330$ is 3.22 . We find that this strengthens the estimated distance from Bailer-Jones et al. (2018).

- Using the same approach for HQ Tau we find a similar

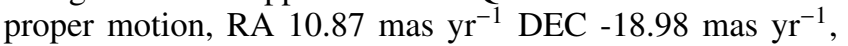
as a small group of 7 stars with an average proper motion

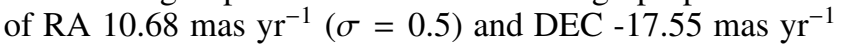
$(\sigma=1.38)$. The spread of parallaxes of these stars range from 6.09 to 6.71 with HQ Tau parallax at 6.2. We see it as likely that these stars are a small co-moving group. This is also strengthened by that the Gaia DR2 parallax lies within $1-\sigma$ of the parallax determined from very-long-baselineinterferometry (VLBI) which associates HQ Tau with the HP Tau/G2 group (Rizzuto et al. 2020). HQ Tau association with this group further strengthens the parallax and we therefore use the distance according to Bailer-Jones et al. (2018) despite of the large RUWE.

- The distance for RY Tau differs greatly between Gaia DR1 and DR2 measurements (corresponding to $176 \mathrm{pc}$ and 442pc respectively). The previous Hipparcos measurement puts RY Tau at a distance of $133 \mathrm{pc}$. The inverted EDR3 parallax suggests that the distance is 138 pc but the RUWE is still very high ( 13). Garufi et al. (2019) argues using the proper motion of RY Tau that it most likely being a member of the Taurus star forming region and that a distance based on the inverted Hipparcos parallax of 133 pc is likely correct. We therefore use the Hipparcos distance for RY Tau.

- Due to the similarity between the Gaia DR2 and Hipparcos parallax for HT Lup, 6.48 and 6.29 mas respectively, we decide to use the value from Bailer-Jones et al. (2018).

- GW Ori lies in the $\lambda$ Ori association and comparing the distance of the association, $403_{-8}^{+13} \mathrm{pc}$ with that of Bailer-Jones et al. (2018), 398 $8_{-10.1}^{+10.6} \mathrm{pc}$, strengthens the Gaia measurement despite the high RUWE. We have decided to use the Gaia based distance.

- EZ Ori was identified by radial velocity as a member of ONC-23 group of stars $\left(399_{-23.4}^{+26.5} \mathrm{pc}\right.$ ) (Kounkel et al. 2018). This confirms the distance from Bailer-Jones et al. (2018) $\left(361_{-33.3}^{+40.7}\right)$ pc.

- The stars Ass Cha-T2 21 and Ass Cha-T2 54 are members of the Chameleon I molecular could. The estimated distances from the Gaia parallax by Bailer-Jones et al. (2018), $164.77_{-3.77}^{+3.95}$ and $202.95_{-16.18}^{+19.19}$ respectively, both overlap with the determined distance to Chameleon I 179 $9_{-10}^{+11}$ (Voirin et al. 2018). We therefore use the distances in Bailer-Jones et al. (2018) for both stars.

- PR Ori is a member of the Lynds 1641 molecular cloud (Mader et al. 1999). A distance determination to Lynds 1641
Table B.1. Stellar parameters following the evolutionary track of a 2 $\mathrm{M}_{\odot}$ PMS star (Siess et al. 2000)

\begin{tabular}{lcccc}
\hline \hline$T_{\text {eff }}$ & $L_{*}$ & $R_{*}$ & $\begin{array}{l}\log \mathrm{g} \\
\mathrm{K}\end{array}$ & $\begin{array}{c}R_{\text {inner }} \\
L_{\odot}\end{array}$ \\
$R_{\odot}$ & $\mathrm{cm} / \mathrm{s}^{2}$ & $\mathrm{AU}$ \\
\hline 4910 & 6.90 & 3.44 & 3.67 & 0.184 \\
5560 & 7.94 & 2.89 & 3.82 & 0.197 \\
6280 & 16.50 & 3.26 & 3.71 & 0.284 \\
7275 & 22.40 & 2.85 & 3.83 & 0.331 \\
9080 & 18.49 & 1.68 & 4.29 & 0.301 \\
\hline
\end{tabular}

was made by Yan et al. (2019) using Gaia DR2 parallaxes. The cloud distance $408_{-4}^{+4} \mathrm{pc}$ is slightly larger than that derived by Bailer-Jones et al. (2018) ( $374 \mathrm{pc})$. We choose to adapt the cloud distance rather then the Gaia derived distance as the distance to PR Ori.

- The parallax of T Tau was measured by Galli et al. (2018) using the VLBI indicating a distance of $148.7( \pm 1.0)$ pc which corresponds well to the Gaia based distance of $143.74_{-1.21}^{+1.22}$ pc.

- For 3 stars no Gaia DR2 parallaxes were available and we searched the literature for distances. V2149 Ori are a member of the Orion Nebula Complex and we used the distance $388 \pm 5$ pc (Villebrun et al. 2019). For UX Tau we used the distance given in Akeson et al. (2019), 139.4 \pm 1.96 pc. And finally for HD 288313A, located in Lynds 1622 at a distance of $418 \pm 17$ (Zucker et al. 2020).

\section{Appendix B: Model SEDs}

A key difference between the classical HAeBe stars and the precursors we are identifying in this paper it the temperature of the start itself. In order to check what difference in the measured disk quantities is down to only this change, and how this compares to the effects of a variation in the scale height of the inner disk, we are computing a simple grid of SEDs. For this purpose, we consider a $2 M_{\odot}$ PMS star using a calculated model SEDs for a passively irradiated disk using stellar parameters taken from the Siess et al. (2000) evolutionary tracks (see table B.1). We use the temperature range between $T_{\text {eff }}=4900 \mathrm{~K}$ and $T_{\text {eff }}=9000 \mathrm{~K}$. We use the Monte Carlo radiative transfer code MCMax (Min et al. 2009), and the DIANA model set-up (Woitke et al.2019) to calculate the SEDs (table B.2. We fix the inner rim dust temperature to $1500 \mathrm{~K}$, which means that the disk inner radius increases with increasing stellar luminosity as the star approaches the Zero Age Main Sequence. We ignore the possible effects of an evolving dust grain population, such as grain settling that may vary in time, inward drift, gaps, holes and planet formation on the dust distribution. We chose two values for the disk scale height at 0.4 $\mathrm{AU}(0.041$ and $0.08 \mathrm{AU})$ to study the effect of the inner disk scale-height on the resulting SED. We derive model values for the $\left[F_{30} / F_{13.5}\right]$ flux ratio and the $7 \mu$ m excess.

Figure $\mathrm{B}$ shows our small model grid, for which $7 \mu$ m excess values increase from 2.7 to 4.6 magnitudes as $T_{\text {eff }}$ increases from 4910 to $9080 \mathrm{~K}$. Increasing the $0.4 \mathrm{AU}$ disk scale height from 0.041 to $0.08 \mathrm{AU}$ changes these numbers to 3.6 and 5.6 magnitudes, respectively. The $\left[F_{30} / F_{13.5}\right]$ remains relatively constant at values between 1.7 and 2.1 for all models. We conclude that an increase in $T_{\text {eff }}$ results in a blueward shift of the stellar SED and therefore an increase in $7 \mu \mathrm{m}$ excess of about 2 magnitudes. There seems no need for changes in the inner disk structure to explain the difference in the maximum $7 \mu \mathrm{m}$ excess between IMTT and HAeBe stars. At the same time, these (gapless) mod- 
Table B.2. Disk parameters used for the evolution model SED.

\begin{tabular}{lc}
\hline \hline parameter & value (unit) \\
\hline$R_{\text {inner }}$ & see table A.1 \\
$R_{\text {tap }}$ & $100 \mathrm{AU}$ \\
$R_{\text {out }}$ & $475 \mathrm{AU}$ \\
$M_{\text {dust }}$ & $2.18 \mathrm{E}-04 M_{\odot}$ \\
gas2dust & 100 \\
$\epsilon$ & 0.66 \\
$\gamma_{\text {exp }}$ & 1.34 \\
$\beta$ & 1.095 \\
sh & $0.041 \mathrm{AU}$ \\
$R_{\text {sh }}$ & $0.4 \mathrm{AU}$ \\
amin & $5.0 \mathrm{E}-02 \mu \mathrm{m}$ \\
amax & $3.0 \mathrm{E}+03 \mu \mathrm{m}$ \\
apow & 3.50 \\
$\alpha_{\text {settle }}$ & 0.01 \\
\hline
\end{tabular}

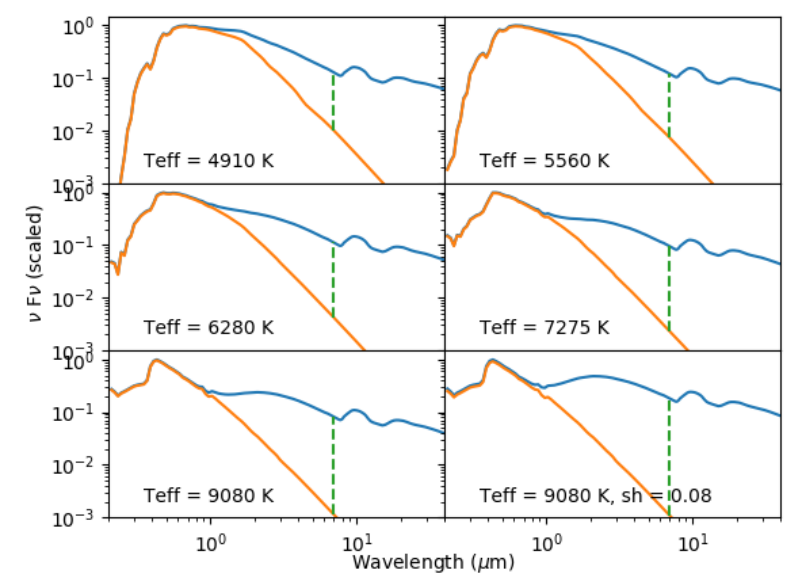

Fig. B.1. SEDs of a $2 M_{\odot}$ PMS star surrounded by a passively heated gas/dust disk. We use a range of luminosities and temperatures (see table B.1) representative of the evolution along a Siess et al. (2000) evolutionary track. The vertical flux scale is normalized to its maximum value for each model. Disk parameters are listed in table B.2 The dashed line indicates the $7 \mu \mathrm{m}$ excess point. The lower right panel shows the effect of increasing the disk scale height at $0.4 \mathrm{AU}$ from 0.041 to $0.08 \mathrm{AU}$, for the $T_{\text {eff }}=9080 \mathrm{~K}$ model.

els, as expected, do not account for the wide range in observed $\left[F_{30} / F_{13.5}\right]$. We refer to Acke et al. (2009) for a full discussion on the effect of model parameters on the $\left[F_{30} / F_{13.5}\right]$ flux ratio.

\section{Appendix C: Individual sources}

In this section we discuss the gas rich disk of stars in the sample which we have found spatially resolved data in scattered light or at millimeter wavelengths in published literature. For each star we check if the spatially resolved data support the Group I or Group II classification based on the SED (section 5). In scattered light and at millimeter wavelengths the Group I disks can be identified having large disk cavity $(\geq 5 A U)$ while Group II disks are smaller continuous disks lacking large cavities (Garufi et al. 2017).

\section{Appendix C.1: $L k H \alpha 330$}

The first detection of the disk was made in IRAS survey data (Weintraub 1990, Weaver \& Jones 1992). The disk has an inner cavity first detected by Brown et al. (2007). Using sub-millimeter imaging the cavity was determined to be $\sim 50-70$ AU (Brown et al. 2009; Andrews et al. 2011) with the outer disk extending to $125 \mathrm{AU}$ (Brown et al. 2009). This is consistent with our SED classification for the disk as a Group I source. Isella et al. (2013), using millimeter-wave interferometry found a lopsided ring of $\sim 100$ AU in the $1.3 \mathrm{~mm}$ dust continuum suggesting the cause to be planet-disk interaction. Observations with the SUBARU in $\mathrm{H}$-band and at $0.87 \mathrm{~mm}$ revealed a spiral structure in the disk (Akiyama et al.2016) explained by the possibility of an unseen planet companion in the disk. Revisiting the star with SUBARU Uyama et al. (2018) found the gap in scattered light to be $\sim 54$ AU also clearly detecting the spiral arms.

\section{Appendix C.2: RY Tau}

An active X-ray source (Skinner et al. 2016) with a visible jet (Agra-Amboage et al. 2009; Garufi et al. 2020) RY Tau has a spectral slope of a Group II disk. ALMA observations reveal a 60 AU disk with a small central cavity two rings (at $\sim 18 \mathrm{AU}$ and $\sim 50$ AU) a gap ( 43 AU) (Pinilla et al. 2018; Long et al. 2018) while Francis \& van der Marel (2020) report an inner cavity of 27 AU.In H-band observations with SUBARU a disk similar in size to the ALMA observations are detected but the cavity, rings and gap are not detected (Takami et al.2013).

\section{Appendix C.3: UX Tau}

UX Tau is a triple system with components A, B and C. The B component also is a binary. The disk around UX Tau A based on its SED is a Group Ia disk. Already by Tanii et al. (2012) classified as pre-transitional. The disk is strongly polarized, geometrically thin and extends to 120AU (Tanii et al. 2012). An inner cavity was detected by Espaillat et al.(2007) and later determined to a size of 25-30AU (Andrews et al. 2011, Tanii et al. 2012, Pinilla et al. 2014) consistent with our classification. The disk has a spiral structure visible both in scattered light and in gas continuum (Zapata et al. 2020; Ménard et al. 2020). The spiral arms likely comes from tidal interaction with UX Tau C towards which one of the arms also extend (Zapata et al. 2020). Zapata et al. further also detects a disk around UX Tau C, material they suggest is probably coming from the interaction with the disk of the A component.

\section{Appendix C.4: HQ Tau}

The dusk around HQ Tau was first classified as a possible debris disk (Andrews \& Williams 2005) later reclassified as a transitional disk (Furlan et al. 2009; Luhman et al. 2010). ALMA observations of the disk (Long et al. 2019) reveals a compact disk with indications of dust depletion towards the inner disk. Our classification as a Group IIa disk fit well with this observation.

\section{Appendix C.5: SU Aur}

Hartmann et al. (2005) using IRAC was fist to detect the infrared excess of SU Aur. Akeson et al. (2005) determined the inner diameter of the dusty disk to be $\sim 0.2 \mathrm{AU}$ based modeling using the observations of 2MASS J, $\mathrm{H}$ and $\mathrm{K}$ band. The disk was studied with both ALMA (de Leon et al. 2015) and SUBARU (Uyama 
et al. 2017; Akiyama et al. 2019) which shows the disk to be disturbed and having a long tail-like structure extending a thousand AU possibly caused by the interaction of a flyby by a sub stellar companion or the ejection of a small object from the disk. Labdon et al. (2019) using the CHARA array performed interferometric observations of SU Aur. They found the disk starting at $0.15 \mathrm{AU}$ extending out to $100 \mathrm{AU}$ with an inclination of $50^{\circ}$. Based on the SED we find this disk to be a Group I source.

\section{Appendix C.6: HD 34700}

HD 34700 is a multiple system where the A component a intermediate binary system, $2 M_{\odot}+2 M_{\odot}$ with a separation of 0.69 AU. (Arellano Ferro \& Giridhar 2003, Sterzik et al. 2005, Monnier et al. 2019). The separation of the B an C components to the A component is 5.18" and 9.17" respectively (Sterzik et al. 2005). HD 34700A was imaged by the Gemini Planet Imager, GPI, (Monnier et al. 2019) which showed a very prominent transition disk inclined at $41^{\circ}$, with a series of spiral arms, surrounding the binary (Monnier et al. 2019). This is consistent with our classification from the SED as a Group I disk. The disk as a large cavity and begins at $175 \mathrm{AU}$ from the center of the system and extends out to $500 \mathrm{AU}$. The cavity is too large to be a consequence of only the two stellar components and could be the effect of a massive planet companion (Monnier et al.2019).

\section{Appendix C.7: GW Ori}

GW Ori s a triple system (Berger et al. 2011) consisting of a spectroscopic binary (GW Ori AB) with a separation of $\sim 1 \mathrm{AU}$ (Mathieu 1994) and a C component, separated $\sim 8 \mathrm{AU}$ (Berger et al. 2011). The disk is a circumtriple disk with a dust component extending to $\sim 400 \mathrm{AU}$ and a gas component extending to $\sim 1400$ AU. The disk is gapped at 25-55 AU (Fang et al. 2014) and spatially resolved ALMA observations shows it to have three dust rings at 46, 188 and $338 \mathrm{AU}$ (Bi et al.2020), the latter the largest dust ring presently known in a protoplanetary disk. Our Group I SED classification fit well with this data. The CO kinematics of the disks suggest that there is a miss-alignment of the inner disks spin axis in respect to the outer disk plane (Bi et al. 2020).

\section{Appendix C.8: V2149 Ori}

V2149 is a known binary star (Köhler et al. 2006), G0+F7 (Daemgen et al. 2012). Kim et al. (2013) classified the disk, based on that the SED was indicative of a central cavity, as transitional and estimated the radius of the disk to 138AU. We find V2149 Ori to be a Group II disk but the uncertainty of the spectral index puts it in the border between Group I and Group II disks. Recent scattered light observations with the SPHERE instrument (Valegard et al. paper in preparation) do not detect any disk. This either means the disk is small or self-shadowed suggesting the Group II classification to be correct.

\section{Appendix C.9: CR Cha}

The first detection of a disk around CR Cha was made by Henning et al. (1993) that classified the disk as Lada Class II. Espaillat et al. (2011) found that the IRS spectra best fitted a pretransitional disk model which is consistent with our classification. A cavity in the disk was suggested by Pinilla et al. (2014) using SED modeling. Varga et al. (2018) used interferometric fitting from MIDI observations to derive an inner disk radius of $\sim 1.3$ AU. Observations by Kim et al. (2020) with ALMA Band 6 show no cavity, instead a gap in the dust continuum at $\sim 90 \mathrm{AU}$ with a width of $\sim 8 \mathrm{AU}$ and a dust ring at $\sim 120 \mathrm{AU}$. The SED of $\mathrm{CR}$ Cha has a spectral slope that places it as a Group IIa source witch is consistent with this continuous inner disk.

\section{Appendix C.10: DI Cha}

DI Cha is a quadruple system with two binaries, one with a G2 and M6 star (the latter possibly a brown dwarf), and the second binary consisting of two M5.5 dwarfs (Schmidt et al. 2013). The angular separation between the two sets of binary stars is 4.6 arcsec. Hendler et al. (2020) find an upper limit of 0.12 arcsec from ALMA continuum imaging (90 per cent light radius) for the mm continuum emission centered on the $\mathrm{G}$ star, and Menu et al. (2015) derive a size of 14.1 milli-arcsec from N-band interferometry. The ALMA and MIDI data taken together suggest a compact disk. Varga et al. (2018) from N-band interferometry find that the inner disk size is not compatible with a continuous disk, but may contain a disk gap on spatial scales of AU. The available imaging is consistent with a classification as a GII source.

\section{Appendix C.11: CV Cha}

CV Cha is a visual binary with an M1 companion at a distance of 11.4 arcsec (Reipurth \& Zinnecker 1993). Hendler et al. (2020) resolve the disk in $\mathrm{mm}$ dust continuum with ALMA and find a size of 0.14 arcsec ( 90 per cent light radius). Menu et al. (2015) resolve the inner disk in the $\mathrm{N}$ band and find a half light radius of 6.1 milli-arcsec. The photosphere of CV Cha is heavily veiled, with the accretion shock covering $20-40 \%$ of the stellar surface (Herczeg \& Hillenbrand 2014). This may introduce some uncertainty in the derived stellar parameters. The available spatial information is consistent with a classification as a GII source.

\section{Appendix C.12: HD 135344B}

HD135344B is a visual binary with the A0 star HD135344A (angular separation 21 arc seconds). Many studies are devoted to the geometry of its circumstellar disk. Andrews et al. (2011) spatially resolve the disk at mm wavelengths using the SMA and find a dust cavity with a radius of 46 AU. Muto et al. (2012) resolve the disk in scattered light and found two spiral arms at 70 AU from the star. Garufi et al. (2013) used NACO at the VLT to measure the radius of the dust in scattered light (probing small grains in the disk surface) and found a radius of $28 \mathrm{AU}$, much smaller than the $46 \mathrm{AU}$ found for the large mid-plane grains. Stolker et al. (2016) and Stolker et al. (2017) find time variable shadows cast on the outer disk, probably resulting from changes in the inner disk structure. van der Marel et al. (2016) resolved the mm dust into a ring at $50 \mathrm{AU}$ and an asymmetric structure at $70 \mathrm{AU}$ (see also Cazzoletti et al. (2018)). These observations convincingly show that HD135344B is a transitional disk (GI), in which planet formation is the likely cause of the observed disk geometry.

\section{Appendix C.13: HT Lup}

HT Lup is a triple system, HT Lup A, B and C. We classify the disk as a Group II which is consistent with DSHARP ALMA continuum images that show a small disk with spiral arm struc- 
ture around the A component that spans roughly 30AU (Andrews et al. 2018; Kurtovic et al. 2018). The B and C component have disks of 5 and 9 AU respectively (Kurtovic et al. 2018). The disk has also been observed by SPHERE (Garufi et al. 2020) and the scattered light signal is consistent with the disk being observed by ALMA for HT Lup A. The disks around the B and C components are not resolved in the scattered light images, neither is the spiral arm structure around the primary component.

\section{Appendix C.14: HD 142666}

DSHARP ALMA continuum images of HD 142666 reveal the presence of relatively narrow rings at distances between 6 and $40 \mathrm{AU}$, and an outer radius of about $90 \mathrm{AU}$ (Andrews et al. 2018; Huang et al. 2018a). The innermost 6 AU seem devoid of large, cold grains (Rubinstein et al. 2018). In scattered light the disk is detected although weakly (Garufi et al. 2017). This star was classified as group II by Meeus et al. (2001). Schegerer et al. (2009) find evidence from near-IR interferometry for a gap at $0.5 \mathrm{AU}$. A comparison of disk size in the near- and midinfrared by Gravity Collaboration et al. (2019) strongly suggests that there is substantial inner disk structure. The classification of HD 142666 as a group II source can still be understood by the fact that the gap dimension is too small to cause a significant imprint on the SED, which was the basis for the classification. The case of HD 142666 illustrates that the group I and group II classification by Meeus et al. (2001), while providing a useful separation between "self-shadowed" disks and (pre-)transitional disks with gaps on scales of 10 AU or more, fails to identify inner disk gaps on scales of AU. Nevertheless, the outer disk does not receive much direct stellar light, as evidenced by the weak signal detected with SPHERE (Garufi et al.2017).

\section{Appendix C.15: HD 142527}

HD 142527 is perhaps the prototypical GI disk source with a large ( 130 AU) gap (Fukagawa et al. 2006; Fujiwara et al. 2006; Ohashi 2008, Casassus et al. 2013; Avenhaus et al. 2014; Rodigas et al. 2014). The prominent shadows detected in scattered light are explained by a highly inclined inner disk (Marino et al. 2015). SMA and ALMA data reveal a horseshoe distribution of dust in the outer disk, usually interpreted as a dust trap (Ohashi 2008; Casassus et al. 2013); see (van der Marel et al. 2013). A stellar mass companion was detected close to the outer radius of the inner disk (Biller et al.|2012; Lacour et al.|2016), which itself is surrounded by an accretion disk. Our SED based classification is confirmed by these observations.

\section{Appendix C.16: Haro 1-6}

Haro 1-6 is a Group I disk based on its SED with one of the highest $\left[F_{30} / F_{13.5}\right]$ in the sample. Images in scattered light taken by SPHERE only marginally detect a disk signal around the edges of the coronograph (Garufi et al. 2020) with several bright filaments extending around the star. Garufi et al. (2020) suggest that these filaments does not have anything to do with the formation of Haro 1-6. No mm emission is detected around Haro 1-6 (Cieza et al.2019). Loinard et al. (2008) suggests Haro 1-6 to be a spectroscopic binary. The PAH emission could have its source either from the strong far-UV and X-ray radiation coming from the binary excites the PAHs in a disk in its last stages of clearing or from a small scale photodissociation region (Jensen et al. 2009).

\section{Appendix C.17: EM*SR 21}

SR21, at a distance of $137.86_{-1.07}^{+1.10}$ pc (Gaia Collaboration et al. 2018) has a binary companion in a wide orbit (Barsony et al. 2003). It might also be a compact binary with the projected distance of about 0.1" (Eisner et al. 2009). SR21 has been classified as a transition disk already based on the shape of the spectrum seen by Spitzer (Brown et al. 2007). Andrews et al. (2011) and Pérez et al. (2014) showed the presence of a large cavity in submm continuum emission, again consistent with a transitional disk. Recently, Muro-Arena et al. (2020) presented combined data from ALMA in Band 3 and SPHERE polarimetric images in the $\mathrm{H}$ band showing a large cavity, a bright ring peaking at 53 $\mathrm{au}$, and spiral structure visible in the scattered light observation inside the main ring, making SR21 a rather unique object. They also show the presence of a kinked spiral connecting the inner and outer disk, matching hydrodynamical predictions of a planet carving the gap and pinpointing its likely position.

\section{Appendix C.18: AK Sco}

AK Sco is a double lined spectroscopic binary Andersen et al. (1989) comprised of a F6+F6 couple with a separation of $\sim 0.16$ AU (Anthonioz et al. 2015). The circumbinary disk around AK Sco was detected first time by Jensen et al. (1996). The overall shape of the SED points towards a Group II disk, but with its steep mid-infrared spectral slope, with $\left[F_{30} / F_{13}\right] \sim 3.3$, our criteria classify it as a Group Ia disk. It has a strong silicate feature (see figure 5) that requires either a different dust opacity or an extreme grain distribution. It is one of the smallest disks detected in polarized light (Garufi et al. 2017). The inner rim of the disk is located at $\sim 0.58 \mathrm{AU}$ (Anthonioz et al. 2015) The polarized light observations show the radius of the disk to be $\sim 13-40$ AU (Janson et al. 2016) with sharp features either from an eccentric ring or a spiral arm structure and confirms the compact nature $\sim 14$ AU in previous observations by ALMA (Czekala et al. 2015). 